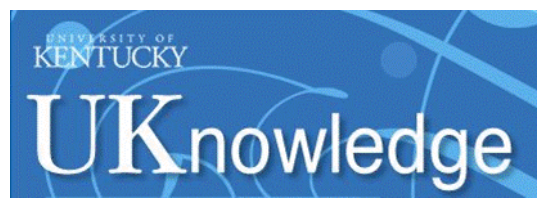

University of Kentucky

UKnowledge

\title{
The Chemical Evolution of QSOs and the Implications for Cosmology and Galaxy Formation
}

Fred Hamann

The Ohio State University

Gary J. Ferland

University of Kentucky, gary@uky.edu

Follow this and additional works at: https://uknowledge.uky.edu/physastron_facpub

Part of the Astrophysics and Astronomy Commons, and the Physics Commons

Right click to open a feedback form in a new tab to let us know how this document benefits you.

\section{Repository Citation}

Hamann, Fred and Ferland, Gary J., "The Chemical Evolution of QSOs and the Implications for Cosmology and Galaxy Formation" (1993). Physics and Astronomy Faculty Publications. 168.

https://uknowledge.uky.edu/physastron_facpub/168

This Article is brought to you for free and open access by the Physics and Astronomy at UKnowledge. It has been accepted for inclusion in Physics and Astronomy Faculty Publications by an authorized administrator of UKnowledge. For more information, please contact UKnowledge@lsv.uky.edu. 


\section{The Chemical Evolution of QSOs and the Implications for Cosmology and Galaxy Formation}

Digital Object Identifier (DOI)

http://dx.doi.org/10.1086/173366

Notes/Citation Information

Published in The Astrophysical Journal, v. 418, no. 1, p. 11-27.

(C) 1993. The American Astronomical Society. All rights reserved.

The copyright holder has granted permission for posting the article here.

This article is available at UKnowledge: https://uknowledge.uky.edu/physastron_facpub/168 


\title{
THE CHEMICAL EVOLUTION OF QSOS AND THE IMPLICATIONS FOR COSMOLOGY AND GALAXY FORMATION
}

\author{
FRED HAMANN ${ }^{1}$ \\ Department of Astronomy, The Ohio State University, 174 West 18th Avenue, Columbus, OH 43210-1106 \\ AND \\ GARY FERLAND \\ Department of Physics and Astronomy, University of Kentucky, Lexington, KY 40506-0055. Internet: gary@asta.pa.uky.edu \\ Received 1993 January 27; accepted 1993 May 26
}

\begin{abstract}
We examine the chemical evolution of QSO broad-line gas by applying spectral synthesis and chemical enrichment models to the $\mathrm{N} v / \mathrm{C}$ IV and $\mathrm{N}$ V/He II emission-line ratios. The models indicate that BLR metallicities are typically $\sim 1$ to perhaps $\gtrsim 10$ times solar. The enrichment must occur in $\lesssim 1$ Gyr for sources where the redshift is $\gtrsim 3$ (if $q_{0}=\frac{1}{2}$ ). The higher metallicity QSOs require star formation favoring massive stars (compared to the Galactic disk). These results imply that extensive evolution usually occurs before the QSOs become observable. Our models of the evolution are equivalent to models proposed for elliptical galaxies and for the bulges of disk galaxies. We conclude that the QSO phenomenon is preceded by vigorous star formation, exactly like that expected in massive, young galactic nuclei.

The observed $\mathrm{N} \mathrm{v/C} \mathrm{IV} \mathrm{and} \mathrm{N}$ v/He II ratios can be several times larger in sources with high redshift and high luminosity. Systematically different physical conditions could contribute to these trends, but they could also result entirely from higher metallicities in the higher redshift/luminosity objects. We suggest that the high metallicities are related to higher QSO (and/or host galaxy) masses at large redshifts. This implies a massmetallicity relation in QSOs analogous to the well-known relationship in nearby ellipticals. The trend with luminosity also suggests that metallicity differences can influence the observed "global Baldwin effect."

The evolution models predict a $\sim 1$ Gyr delay in the Fe enrichment due to Type Ia supernovae. The timescale for this delay is fixed by the (albeit uncertain) lifetimes of SN Ia precursors and is not sensitive to the IMF or star formation rates. The expected $\sim 1$ Gyr delay could therefore be used as a clock to constrain QSO ages if accurate Fe abundances are measured. Age constraints could in turn constrain the cosmology (i.e., $q_{0}$ ) when applied to high-redshift sources. One-zone photoionization models suggest that the delayed rise in $\mathrm{Fe}$ should be observable in, for example, the ratio of $\mathrm{UV} \mathrm{Fe} \mathrm{II/Mg} \mathrm{II} \mathrm{emission} \mathrm{lines.}$

Most of the evolution models also predict $\mathrm{Fe}$ overabundances after $\sim 1-2 \mathrm{Gyr}$, with $\mathrm{Fe} / \mathrm{O}$ and $\mathrm{Fe} / \mathrm{Mg}$ up to several times solar. This overabundance might explain the strong Fe II emission observed in many QSOs and active galactic nuclei.
\end{abstract}

Subject headings: cosmology: theory - galaxies: abundances - galaxies: evolution - line: formation quasars: general

\section{INTRODUCTION}

The broad emission lines of QSOs offer direct probes of the chemical composition and enrichment history of the gas. Unfortunately the line strengths are not sensitive to the total metallicity (Davidson 1973; Ferland \& Hamann 1993; Baldwin et al. 1993), but some of the line ratios are sensitive to the relative abundances, and these can be used to constrain both the metallicity and evolution parameters. The abundance ratios are tied to the evolution because the elements form by different processes and on different timescales (cf. the review by Wheeler, Sneden, \& Truran 1989). For example, the O, Ne, $\mathrm{Mg}$, and $\mathrm{Ca}$ enrichment is very rapid because these elements derive mostly from massive star supernovae (e.g., Type II). The $\mathrm{C}$ enrichment is delayed somewhat, because it forms partly in the envelopes of longer lived intermediate-mass stars. Similarly, $\mathrm{Fe}$ is delayed by the contribution from Type Ia supernovae, which also have intermediate mass precursors. The $\mathrm{N}$ enrichment is also delayed because it is at least partly a

\footnotetext{
${ }^{1}$ Present address: Center for Astrophysics and Space Sciences, University of California-San Diego, Mail Code 0111, La Jolla, CA 92093-0111. Internet: fred@cass157.ucsd.edu.
}

"secondary" element, formed by CNO burning in stellar envelopes. By contrast, the $\mathrm{He}$ abundance is mostly primordial and changes relatively little as the "metals" increase by orders of magnitude.

Previous estimates of abundances in QSO broad-line regions (BLRs) relied on measurements of several intercombination lines (Shields 1976; Davidson 1977; Osmer 1980; Gaskell, Shields, \& Wampler 1981; Uomoto 1984). Those lines are not only weak and undetected in most sources, but they are also subject to collisional deexcitation at densities above $\sim 10^{10}$ $\mathrm{cm}^{-3}$. The results are therefore limited to selected objects and compromised by the fact that both the densities and fluxes are probably at least a decade larger than previously believed (see Ferland et al. 1992 and the review by Peterson 1993). As a result, the intercombination lines might all be controlled by their saturation densities. Nonetheless, the early studies suggest that the relative $\mathrm{N}$ abundance is usually equal to, or several times above, solar. Recently Hamann \& Ferland (1992, hereafter HF92) extended this analysis to a much larger sample of objects by modeling the strong lines C IV $1550 \AA$ (hereafter C IV) and $N$ v $1240 \AA$ ( N v). In that work we estimated N/C 22 times solar and metallicities $Z \gtrsim 3 Z_{\odot}$ in redshift $>4$ QSOs, 
and a wider range of abundances, perhaps reaching $Z \gtrsim 10$ $Z_{\odot}$, in the large sample of QSOs with redshift $\sim 2-4$. Large metallicities at the highest redshift require vigorous star formation, with most of the evolution occurring in $\lesssim 0.5$ Gyr if $q_{0} \approx$ $\frac{1}{2}$ or $\$ 2.0 \mathrm{Gyr}$ if $q_{0} \approx 0$. The evolution models of HF92 also indicate that initial mass functions (IMFs) favoring high-mass stars are needed to achieve the high $Z$ 's. The abundances and evolution parameters are similar to those derived for giant elliptical galaxies and for the bulges of disk galaxies (Arimoto \& Yoshii 1987; Matteucci \& Tornambè 1987; Bica 1988; Bica, Arimoto, \& Alloin 1988; Rich 1988; Matteucci \& Brocato 1990; Köppen \& Arimoto 1990). Therefore the QSO results are not surprising; they are consistent with QSOs residing in massive galactic nuclei.

Line variability studies (cf. Clavel et al. 1991; Peterson 1993) of active galactic nuclei (AGNs) and estimates of the $\mathrm{N}^{+4}$ and $\mathrm{C}^{+3}$ ionization structures (e.g., Ferland \& Hamann 1993) both indicate that $\mathrm{N} v$ forms closer to the central source than $\mathrm{C}$ IV. One-zone photoionization models (HF92) can still be used, but the $\mathrm{N} / \mathrm{C}$ abundances derived from $\mathrm{N} v / \mathrm{C}$ IV are sensitive to the geometry and to possible gradients in the physical conditions across the BLR. For example, regions with limited column density might produce $\mathrm{N} v$ without $\mathrm{C}$ IV (Davidson \& Netzer 1979), leading to overestimates of N/C. In addition, constraining the metallicity and enrichment history of the gas based on just $\mathrm{N} / \mathrm{C}$ is limited because the detailed evolution of $\mathrm{N}$ and $\mathrm{C}$ are model dependent. Nonetheless, the qualitative results for enhanced $\mathrm{N}$ and high $Z$ (relative to solar) hold for "standard" chemical enrichment schemes and for a wide range of densities and ionizing fluxes in the spectral synthesis (HF92).

Large $\mathrm{N}$ abundances were confirmed recently by analysis of the ratio $\mathrm{N} v$ to $\mathrm{He}$ II $1640 \AA$ (He II) in several QSOs with redshift $\gtrsim 2$ (Ferland \& Hamann 1993; Baldwin et al. 1993). This line ratio is a more robust abundance indicator than $\mathrm{N} \mathrm{v}$ / C IV because $\mathrm{N}^{+4}$ resides within the $\mathrm{He}^{++}$ionization zone (where $\mathrm{He}$ II forms as a recombination line). If $\mathrm{N}^{+4}$ does not fill the $\mathrm{He}^{++}$zone the $\mathrm{N}$ v emission can be weak, but it is not possible to produce $\mathrm{N} v$ without also producing $\mathrm{He}$ II. Thus, $\mathrm{N}$ $\mathrm{v} / \mathrm{He}$ II provides a firm lower limit to $\mathrm{N} / \mathrm{He}$. These results might be considered true estimates rather than just lower limits because roughly "standard" spectral synthesis parameters lead to $\mathrm{N}$ V/He II near its maximum (Baldwin et al. 1993; this work). The combination of N/C and $\mathrm{N} / \mathrm{He}$ abundances also places tighter constraints on $Z$ and the evolution parameters.

In this paper we analyze a large sample of $\mathrm{N} \mathrm{v/He} \mathrm{II} \mathrm{and}$ $\mathrm{N} v / \mathrm{C}$ IV observations and estimate QSO abundance and chemical evolution by requiring self-consistent results for both ratios in simple one-zone models. We use abundances from the evolution models directly in the photoionization/spectral synthesis calculations. We show in detail the effects of parameter variations in the chemical evolution and provide a limited discussion of the parameter sensitivities in the spectral synthesis. The details of the spectral synthesis with various input parameters are discussed by Ferland \& Hamann (1993), Hamann \& Ferland (1993a), and Baldwin et al. (1993). In the present work we also (1) describe the abundance evolution of several elements, including $\mathrm{H}, \mathrm{He}, \mathrm{C}, \mathrm{N}, \mathrm{O}, \mathrm{Mg}$, and $\mathrm{Fe}$, in various enrichment scenarios; (2) demonstrate the utility of $\mathrm{Fe}$ as a "clock" to measure QSO ages and perhaps constrain the cosmology; (3) examine an observed trend for increasing $\mathrm{N} \mathrm{v/C} \mathrm{IV}$ and $\mathrm{N} \mathrm{v} / \mathrm{He}$ II ratios at higher redshifts and higher luminosities in terms of a possible mass-metallicity-redshift correlation; (4) describe the evolution of the stellar luminosities and super- novae rates in a "typical" QSO/giant elliptical galaxy model that reaches high metallicities at high redshift; and (5) calculate the thermal energy from supernovae, as a trigger of galactic winds, to place lower limits on the total masses needed to bind the gas.

Our approach to the evolution is to develop first a reference model of the solar neighborhood in order to fix the nucleosynthesis and test the results against observations of the galaxy. With the nucleosynthesis fixed, the only free parameters are the shape of the IMF and the timescales for star formation and primordial infall (i.e., the mass buildup of the system). We then develop models for the QSOs by changing just these parameters.

Throughout this paper we use the (nonstandard) convention that bracketed quantities such as $[\mathrm{H}],[\mathrm{N}],[\mathrm{Z}],[\mathrm{Fe} / \mathrm{O}]$, etc., and linear mass abundances relative to solar (Grevesse \& Anders 1989). They are defined as mass fractions (or mass fraction ratios for $\mathrm{Fe} / \mathrm{O}$ ) divided by the corresponding mass fractions in the Sun.

\section{CHEMICAL EVOLUTION CALCULATIONS}

\subsection{Methods and Assumptions}

The enrichment calculations differ slightly from HF92 to account for modest convective overshooting in the intermediate-mass stars and include the recent massive star nucleosynthesis results of Weaver \& Woosley (1992). Our formulation of the chemical evolution equations closely follows Talbot \& Arnett (1973), Matteucci \& Greggio (1986), and Matteucci \& Tornambè (1987). The integrodifferential equations are solved using a fifth-order Runge-Kutta method with subroutines adapted from Press et al. (1986). The models describe a closed system assembled by the infall of primordial gas composed of $76 \% \mathrm{H}$ and $24 \% \mathrm{He}$ by mass. The infall rate is described by an exponential with a characteristic decay time. The calculations follow the abundance evolution of all the major elements. The enrichment delays caused by finite stellar lifetimes are included by using the main-sequence lifetimes of Scalo (1986). The models assume the stellar enrichment occurs all at once at the time of the star's death. The ejecta are instantly mixed with the interstellar gas and available for further star formation. Stars form over the mass range $0.087 \leq M_{*} \leq 100$ $M_{\odot}$, although we also consider the effects of raising the lower mass limit to $2.5 M_{\odot}$. For simplicity we use power law IMFs of the form $\Phi \propto M_{*}^{-x}$, with a possible change in slope at $M_{*}=1$ $M_{\odot}$. The IMF is normalized so that

$$
\int_{M_{\min }}^{M_{\max }} \Phi(m) d m=1,
$$

where $M_{\min }$ and $M_{\max }$ are the minimum and maximum masses used in the calculation. In this notation the Salpeter IMF has slope $x=1.35$. We assume the IMF is constant in space and time. We also assume that the stellar birthrate, $\Psi(t)$, scales linearly with the gas density,

$$
\Psi(t)=v G(t),
$$

where $v$ is a constant and $G(t)$ is the gas density at time $t$ normalized to the final total density after all of the accretion has occurred. The choice of a linear scaling with density is arbitrary, but our main results are not sensitive to the form of the birthrate function. They depend only on the timescales for infall and star formation and on the differences that result from changing these quantities. Had we adopted a different func- 
tional form for $\Psi(t)$, we would have simply used different scale factors $(v)$ to match the data and achieve the same enrichment times.

We consider four sites of chemical enrichment: (1) the envelopes of intermediate-mass stars $\left(1 \lesssim M_{*} \lesssim 7.0 M_{\odot}\right)$, (2) Type Ia supernovae (hereafter SN Ia's), (3) the exploding He cores of massive stars as Types II and Ib supernovae (SN II + Ib's), and (4) the envelopes above the He cores of massive stars $\left(M_{*} \gtrsim 7.0 M_{\odot}\right)$. Stars below $\sim 1 M_{\odot}$ do not contribute because their lifetimes are too long. The yields we adopt are consistent with the most recent stellar nucleosynthesis calculations, as described below. They are nonetheless sometimes ad hoc and contrived to produce good agreement between observations and our models of the solar neighborhood $(\S 2.2$ below). Ultimately none of our main results for the QSOs depend on these choices ( $\$ 4.1)$. An unavoidable assumption in our models is that the stellar yields, which are all based on calculations with $Z \leq Z_{\odot}$, remain approximately correct for $Z>Z_{\odot}$

1. Intermediate-mass stars.-Intermediate-mass stars are important contributors of $\mathrm{He}, \mathrm{C}$, and $\mathrm{N}$. These stars have too little mass to ignite $\mathrm{C}$ in their cores and so end their lives as $\mathrm{He}$ or $\mathrm{CO}$ white dwarfs. The yields from their envelopes were estimated by Renzini \& Voli (1981). We consider two cases that include primary plus secondary, and secondary only $\mathrm{N}$ production (their models with $\eta=0.333$ and $\alpha=1.5$ and 0.0 , respectively). "Primary" $\mathrm{N}$ derives from $\mathrm{C}$ and $\mathrm{O}$ produced within the star, while "secondary" $\mathrm{N}$ derives from the $\mathrm{C}$ and $\mathrm{O}$ available when the star formed. The relative contributions from these processes are not well known, but some secondary $\mathrm{N}$ appears in both cases we consider. Another unknown is the amount of core convective overshooting and its affect on the stellar yields. Renzini \& Voli (1981) did not include overshooting, and recent work by Stothers (1991), Stothers \& Chin (1991), and Catellani, Chieffi, \& Straniero (1992) suggests that it is less extensive than some previous claims. However, it appears that even a small amount of overshooting can significantly alter the yields (cf. Maefer \& Meynet 1987, 1989). Also, using more recent opacities in the stellar evolution codes increases the size of the convective core (Stothers \& Chin 1991) and thus mimics this aspect of overshooting (Becker \& Iben 1979; Bertelli, Bressen, \& Chiosi 1985; Maeder \& Meynet 1987) even when overshooting is not present. We have therefore modified Renzini \& Voli's (1981) results to account for the changes expected when modest overshooting occurs. In particular, we adopt an upper mass limit for the intermediate-mass stars of $M_{\text {up }}=7.0 M_{\odot}$. This value is smaller than the $8 M_{\odot}$ used by Renzini \& Voli (1981) without overshoot, but lies within the range $\left(\sim 6.3\right.$ to $\left.\sim 7.0 M_{\odot}\right)$ estimated by Maeder \& Meynet (1989) with overshoot included. Lower values of $M_{\text {up }}$ are expected with either overshooting or the new opacities because of the larger core masses. We also increased Renzini \& Voli's (1981) yields per stellar mass slightly to account for the greater processing expected for overshooting (Greggio \& Tosi 1986; Serranto 1986; Maeder \& Meynet 1989). We did this by expressing Renzini \& Voli's (1981) tabulated yields as fractions of the stellar mass and shifting the mass grid downward by 0.5 $M_{\odot}$. This increased the C production by only $\lesssim 20 \%$ in our solar neighborhood models $(\$ 2.2)$ but had a negligible effect on the other elements. In the case of secondary-only $\mathrm{N}$ production, Renzini \& Voli's $N$ yields. (their $\alpha=0.0$ case) did not produce enough $\mathrm{N}$ in our solar neighborhood model. We therefore used Serrano's (1986) larger secondary $\mathrm{N}$ yields (with overshoot), which combine with the secondary $\mathrm{N}$ from highmass stars (below) to agree well with solar abundances. In the primary + secondary $\mathrm{N}$ case, Renzini \& Voli's $\alpha=1.5$ models produced too much primary $\mathrm{N}$ and not enough $\mathrm{C}$. We therefore used an average of the $\mathrm{C}$ and primary $\mathrm{N}$ yields from their $\alpha=0.0$ and $\alpha=1.5$ cases. Finally, we increased the production of He slightly by using the yields of Serrano (1986). This gave better agreement with observational estimates of the growth of He relative to the metals $(\Delta Y / \Delta Z)$ in the solar neighborhood (see $\S 2.2$ below).

2. Type Ia supernovae.--Type Ia supernovae contribute mostly $\mathrm{Fe}$ and are believed to be important sources of $\mathrm{Fe}$ in our galaxy (cf. Abia, Canal, \& Isern 1991, and the review by Wheeler \& Harkness 1990). They are treated in our models as exploding $\mathrm{CO}$ white dwarfs in close binaries (after Greggio \& Renzini 1983 and Matteucci \& Greggio 1986). We assume the secondary stars do not contribute to the enrichment. The envelopes of the primaries contribute normally, as if they were single stars. The exploding cores of the primaries always have a mass of $1.4 M_{\odot}$. For initial primary masses below $\sim 5 M_{\odot}$, the cores must accrete mass from the secondary to reach $1.4 M_{\odot}$. The explosive yields are taken from Nomoto, Thielemann, \& Yokoi (1984; their model w7). In our models, the lifetime of the binary is determined by the main-sequence life of the secondary (but see Smecker \& Wyse 1991 for discussion). We consider binaries with total masses between 3.0 and $14.0 \mathrm{M}_{\odot}$ as potential SN Ia progenitors. The secondary masses range from 0.9 to $7.0 \mathrm{M}_{\odot}$, which implies lifetimes of $\sim 14.6$ to $\sim 0.06 \mathrm{Gyr}$, respectively (Scalo 1986). The fraction, $A$, of the IMF between 3.0 and $14.0 M_{\odot}$ that produces $\mathrm{SN}$ Ia's is estimated by requiring solar $\mathrm{Fe}$ abundances in the solar neighborhood models below. The fraction we derive, $A \approx 0.1$, agrees with previous estimates based on galactic supernova rates (Matteucci \& Greggio 1986; Matteucci \& François 1989).

3. Types $I I+I b$ supernovae.-The yields from massive stars, $7<M_{*}<100 M_{\odot}$, are divided into the contributions from the envelopes and the He cores. We assume the cores explode as core bounce supernovae of Types Ib or II. The exploding cores are important contributors of $\mathrm{He}$ and all of the major metals, but only stars with initial masses above $\sim 12 M_{\odot}$ contribute significantly to the heavy metal enrichment. For stars with masses between 7 and $12 M_{\odot}$ we adopt the small $\mathrm{C}$ yields estimated by Talbot $\&$ Arnett (1973; their $q_{c}$ parameter), and use $\mathrm{He}$ yields interpolated from the values adopted at $M_{*}=7$ $M_{\odot}$ (above) and $12 M_{\odot}$ (below). For masses between 12 and 40 $M_{\odot}$ we derive core compositions from the recent nucleosynthesis results of Weaver \& Woosley (1992). The corresponding core masses were generously provided by S. E. Woosley (1992; private communication). Above $40 M_{\odot}$ we use an average of the yields given by Arnett (1978) and Woosley \& Weaver (1986), which is roughly consistent with Weaver \& Woosley (1992) at $40 M_{\odot}$ (e.g., in C/O; see below). In this mass range (actually $M_{*} \gtrsim 35 M_{\odot}$ ) we adopt He core masses from Maeder \& Meynet (1987).

Weaver \& Woosley (1992) examined the influence on the yields of the uncertain ${ }^{12} \mathrm{C}(\alpha, \gamma){ }^{16} \mathrm{O}$ reaction rate. Their preferred value is intermediate between the rates used by Woosley \& Weaver (1986) and Arnett (1978) and leads to generally intermediate yields, most importantly of C. In HF92 we used the results of Arnett (1978) because C production given by Woosley \& Weaver (1986) appeared too small for our solar neighborhood models. However, Arnett's (1978) yields of C are almost certainly too large. In particular, for all of the IMFs 
used in this paper they predict $[\mathrm{C} / \mathrm{O}] \sim 0.7$ to 0.8 due to $\mathrm{SN}$ II + Ib's alone. These ratios appear inconsistent with recent observations of $[\mathrm{C} / \mathrm{O}] \sim 0.1$ to 0.2 in galactic halo stars (Abia et al. 1991; Nissan 1990; see also $\S 2.2$ below). In our solar neighborhood models below Arnett's (1978) yields also predict a large ratio of $[\mathrm{C} / \mathrm{O}] \sim 1.7$ in the Sun. The new results of Weaver \& Woosley (1992), on the other hand, produce good agreement with solar abundances and better agreement with the observed delay in the $[\mathrm{C} / \mathrm{O}]$ enrichment $(\$ 2.2)$.

The Fe yields from core bounce supernovae are theoretically uncertain because they require an understanding of the explosive nucleosynthesis. Arnett (1991) estimated Fe yields from observations of SN 1987A (cf. the review by Arnett et al. 1989). For stellar masses below $40 M_{\odot}$, we adopt Fe yields equivalent to Arnett (1991) by converting $20 \%$ of the presupernova Si, S, $\mathrm{Ar}$, and Ca calculated by Weaver \& Woosley (1992) into Fe in the ejecta. Above $40 M_{\odot}$ we apply the same $20 \%$ conversion to the Woosley \& Weaver (1986) yields, adopt Arnett's (1991) Fe production directly with the Arnett's (1978) yields, and use the average of the two as for the other elements.

4. Massive star envelopes.-The envelopes above the $\mathrm{He}$ cores in massive stars produce significant $\mathrm{He}$ and secondary $\mathrm{N}$ via $\mathrm{CNO}$ shell burning. There is no primary $\mathrm{N}$ production in this stellar mass range. ${ }^{1}$ The envelope He production is derived from the tables in Weaver \& Woosley (1992). Their results predict a conversion of $\sim 4 \%$ of the envelope $\mathrm{H}$ into $\mathrm{He}$ at $M_{*}=12 M_{\odot}$ and $\sim 27 \%$ at $40 M_{\odot}$. Above $40 M_{\odot}$ we adopt a constant conversion rate of $27 \%$. For the secondary $N$ production, we convert $75 \%$ of the envelope $\mathrm{C}$ and $\mathrm{O}$ into $\mathrm{N}$. This conversion factor approximates the nucleosynthesis results of Maeder (1983) and Maeder \& Meynet (1987) for $M_{*} \gtrsim 40 M_{\odot}$, and roughly equals the conversion predicted by Serano (1986) at $6 M_{\odot}$ in his models with overshooting. It is also intermediate between the $100 \%$ conversion used by Matteucci (1991) in her galactic disk models, and the calculations of Weaver \& Woosley (1992) which indicate $\sim 20 \%-45 \%$ conversion for $12 \leq M_{*} \leq 40 M_{\odot}$, respectively. In our solar neighborhood models ( $\$ 2.2$ ) the $75 \%$ conversion combines with the $\mathrm{N}$ from intermediate-mass stars (above) to produce solar $\mathrm{N}$ abundances. Although the amount of secondary conversion is uncertain, we note that using $100 \%$ has a negligible effect on the models and much smaller values only reinforce our main results in $\S 4$ (that the large $\mathrm{N} v / \mathrm{C}$ IV and $\mathrm{N} v / \mathrm{He}$ II line ratios in QSOs require highly evolved systems with high metallicities).

\subsection{A Reference Model of the Solar Neighborhood}

We constructed a reference model of the solar neighborhood to fix the nucleosynthesis parameters and test the calculations against observations of the galaxy. The models include all of the key features of more complex descriptions of the solar neighborhood (e.g., Matteucci \& François 1989; Köppen \& Arimoto 1990), but they are not intended to be a complete description of the galactic disk. For example, they cannot account for the observed abundance gradients (cf. the reviews by Pagel \& Edmunds 1981 and Diaz 1989) because of the

\footnotetext{
${ }^{1}$ Nucleosynthesis calculations indicate that some primary $\mathrm{N}$ might be produced by high-mass stars with "zero" initial metallicity (Woosley 1992, private communication). However, the theoretical yields are very uncertain and we did not attempt to include them here. We simply note that a primary $\mathrm{N}$ contribution from massive stars with very low $Z$ would not effect our results concerning the QSOs (§ 4). The important evolution occurs at much higher Z’s.
}

one-zone treatment. Nonetheless, we will show that the models are consistent with all of the essential data on galactic abundances and supernova rates. The models might therefore be a good first approximation to the solar neighborhood evolution.

We adopt an infall (disk formation) timescale of 3.0 Gyr and a present galactic age of 13 Gyr (after Matteucci 1991 and Matteucci \& François 1989). The power-law IMF has a slope of $x=1.1$ for $M_{*} \leq 1 M_{\odot}$ and $x=1.6$ for $M_{*}>1 M_{\odot}$. This IMF consistent with the observed present day mass function (Scalo 1986) and is roughly intermediate between the IMFs used in the galactic disk models of Matteucci \& François (1989) and Köppen \& Arimoto (1990). Note that the slope of 1.6 is slightly steeper than the 1.5 adopted in HF92. The slope $x=1.1$ for $M_{*} \leq 1$, and the magnitude of the stellar birthrate, $v$, are uniquely determined by the requirements that (1) the metallicity at the time of the Sun's formation $(t \approx 8.5 \mathrm{Gyr})$ is solar, and (2) the ratio of the mass in gas, $M_{g}$, to total mass in gas, stars, and stellar remnants, $M_{T}$, at the present epoch $(t=13 \mathrm{Gyr})$ is $g \equiv M_{g} / M_{T}=15 \%$.

The fraction of stars forming binary $\mathrm{SN}$ Ia precursors, $A \approx 0.1$ ( $(2.1)$, leads to a current ratio of SN II + Ib to SN Ia rates of $\sim 2.4$ (at $13 \mathrm{Gyr}$ ), and an average ratio over the life of the galaxy of $\sim 3.8$. These $\mathrm{SN}$ rates are consistent with observational estimates for the galaxy and for late-type spirals in general (Evans van den Bergh, \& McClure 1989; also Wheeler \& Harkness 1990 and references therein). The derived absolute rates depend on the total mass involved. If the mass of gas, stars, and stellar remnants in our galaxy is $M_{T}=10^{11} M_{\odot}$, then the models predict a current SN II + Ib rate of $\sim 2.4$ per $100 \mathrm{yr}$, and a total number of SN II + Ib's over the life of the galaxy of $\sim 6.4 \times 10^{8}$. These numbers are also within the uncertainties of observational estimates (Evans et al. 1989; Arnett, Schramm, \& Truran 1989).

Figures 1 and 2 show the abundance evolution of some of the major elements in the various models discussed in this paper. Each model is labeled in the upper left and the parameters that define the models are listed in Table 1. The IMFs are defined by their power-law indices and the stellar birthrates are described by $v$ from equation $2(\S 2.1)$. The rate of gas consumption is characterized by $t(15 \%)$, the time at which the mass fraction in gas is $g=15 \%$. The solar neighborhood models are M1a and M1b. They are identical except that M1a and all of the " $\mathrm{a}$ " models use only secondary $\mathrm{N}$ production, while M1b and the " $b$ " models include primary + secondary $\mathrm{N}(\S 2.1)$. For convenience Figure 2 shows only the secondary $\mathrm{N}$ models. The models M2-M6 are discussed in $\S 2.3$ below.

In the solar neighborhood models $\mathrm{M} 1$ the ratio $[\mathrm{Mg} / \mathrm{O}]$ is nearly constant because both elements are produced mainly by SN II + Ib's (Fig. 1). The $\mathrm{N}$ abundance is low at early epochs because it is not newly created by massive stars $(\S 2.1)$. The $[\mathrm{N} / \mathrm{O}]$ ratio rises abruptly in $\mathrm{M} 1 \mathrm{~b}$ when the first primary $\mathrm{N}$ is ejected from the intermediate mass stars. In $\mathrm{M} 1 \mathrm{a}[\mathrm{N} / \mathrm{O}]$ rises more gradually because the $\mathrm{N}$ is purely secondary. Unfortunately, it is difficult to choose between these models because the true sources of $\mathrm{N}$ remain uncertain (cf. Wheeler et al. 1989 and references therein). When the "raw" data from galactic dwarf stars (Tomkin \& Lambert 1984) and Galactic and extragalactic $\mathrm{H}$ II regions (Pagel 1985) are examined in terms of the $[\mathrm{N} / \mathrm{O}]$ versus $[\mathrm{O} / \mathrm{H}]$ dependence, there is evidence for mostly secondary $\mathrm{N}$ production near solar metallicities. A primary contribution may be needed to explain the apparent plateau in N/O at very low $Z$ (also Pagel \& Edmunds 1981; Matteucci 1989). However, the large scatter in the $\mathrm{H}$ II region data would 



FIG. 1.-Abundances relative to oxygen and normalized to solar are plotted against age for each of the models listed in Table 1. The models are identified in the upper left of each plot. The curves are identified in the lower right. The abundances convergence toward solar in the solar neighborhood model (M1a), but exhibit large deviations from solar in the other models. 

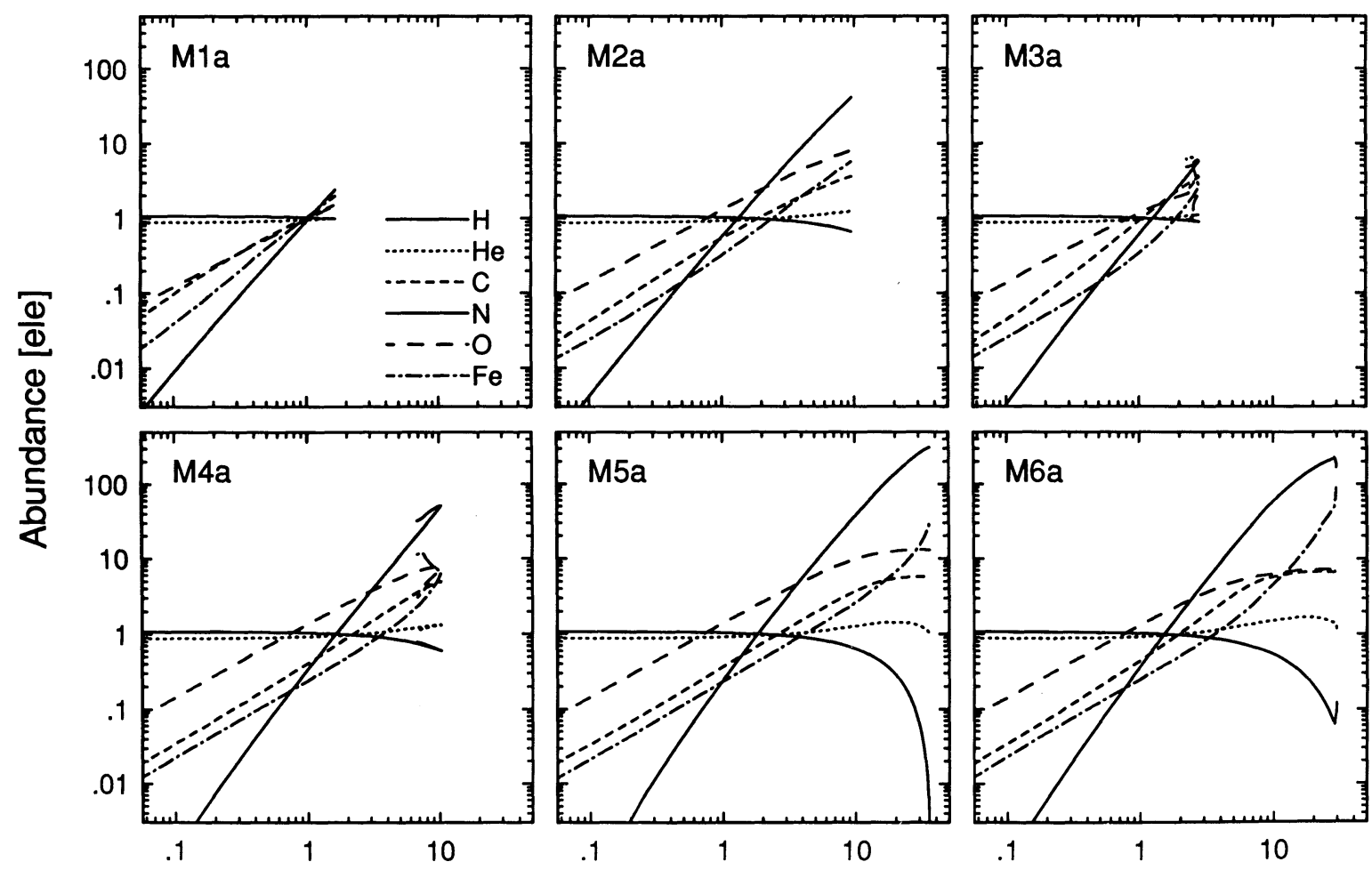

Metallicity [Z]

Fig. 2.-Abundances of several elements normalized to solar are plotted against $Z$ for each of the secondary N models M1a-M6a. The curves are identified in the upper left-hand panel. The abundances converge toward solar at $Z=Z_{\odot}$ in M1a, but deviate substantially from solar in the QSO models M4a-M6a. The curves are double-valued in M3a and M4a because the metallicity declines after the star formation is halted (see Fig. 3 and $\S 2.3$ ). The calculation stops in M5a when the interstellar $\mathrm{H}$ is exhausted.

TABLE 1

Summary of Chemical Evolution Models

\begin{tabular}{|c|c|c|c|c|c|c|}
\hline \multirow[b]{2}{*}{ MODEL } & \multirow[b]{2}{*}{ N Production ${ }^{a}$} & \multirow[b]{2}{*}{$\mathrm{IMF}^{\mathrm{b}}$} & \multirow[b]{2}{*}{$\begin{array}{c}v \\
\left(G y r^{-1}\right)\end{array}$} & \multicolumn{2}{|c|}{ Timescales } & \multirow[b]{2}{*}{ COMMENTS } \\
\hline & & & & $\begin{array}{l}\text { Infall } \\
\text { (Gyr) }\end{array}$ & $\begin{array}{l}t(15 \%) \\
(\mathrm{Gyr})\end{array}$ & \\
\hline M1a ......... & Secondary & $x=1.1$ for $M_{*}<1 M_{\odot}$ & 0.27 & 3.0 & 13.0 & \multirow{2}{*}{ Solar neighborhood } \\
\hline M1b .......... & Primary & $x=1.6$ for $M_{*}^{*}>1 M_{\odot}$ & 0.27 & 3.0 & 13.0 & \\
\hline M2a & Secondary & $x=1.0$ & 0.42 & 3.0 & 13.0 & \multirow{2}{*}{$\begin{array}{l}\text { Solar neighborhood timescales } \\
\text { with "shallow" IMF }\end{array}$} \\
\hline $\mathrm{M} 2 \mathrm{~b} \ldots \ldots \ldots$ & Primary & $x=1.0$ & 0.42 & 3.0 & 13.0 & \\
\hline M3a . & Secondary & $x=1.1$ for $M_{*}<1 M_{\odot}$ & 5.0 & 0.05 & 0.5 & \multirow{2}{*}{$\begin{array}{l}\text { Low-metallicity QSO (?) } \\
\text { (“solar" IMF + short times) }\end{array}$} \\
\hline M3b .......... & Primary & $x=1.6$ for $M_{*}^{*}>1 M_{\odot}$ & 5.0 & 0.05 & 0.5 & \\
\hline M4a ........ & Secondary & $x=1.1$ & 6.7 & 0.05 & 0.5 & \multirow{2}{*}{$\begin{array}{l}\text { High-metallicity QSO (?) } \\
\approx \text { giant elliptical model }\end{array}$} \\
\hline M4b .......... & Primary & $x=1.1$ & 6.7 & 0.05 & 0.5 & \\
\hline M5a .......... & Secondary & $x=1.0, M_{\text {cut }}=2.5 M_{\odot}$ & 7.6 & 0.05 & 1.8 & \multirow{2}{*}{$\begin{array}{l}\text { “Extreme" QSO (?) } \\
\text { (truncated "shallow" IMF) }\end{array}$} \\
\hline M5b .......... & Primary & $x=1.0, M_{\text {cut }}=2.5 M_{\odot}$ & 7.6 & 0.05 & 1.8 & \\
\hline M6a ......... & Secondary & $x=1.6, M_{\mathrm{cut}}=2.5 M_{\odot}$ & 7.6 & 0.05 & 1.3 & \multirow{2}{*}{$\begin{array}{l}\text { “Extreme" QSO (?) } \\
\text { (truncated "solar" IMF) }\end{array}$} \\
\hline M6b ........... & Primary & $x=1.6, M_{\text {cut }}=2.5 M_{\odot}$ & 7.6 & 0.05 & 1.3 & \\
\hline
\end{tabular}

a "Secondary" $\mathrm{N}$ implies secondary only, while " primary" implies primary + secondary (see $\S 2.1)$.

${ }^{b}$ The IMF is a power law with slope(s) $-x$. The mass range is from 0.087 to $100 M_{\odot}$ except in M5 and M6, where the lower mass cutoff is $M_{\text {cut }}=2.5 M_{\odot}$. 
suggest very different amounts of primary $\mathrm{N}$ in different sources (Pagel \& Edmunds 1981). It is also not clear that the data from present-day $\mathrm{H}$ II regions can be interpreted in terms of an evolutionary sequence in [N/O] (Matteucci 1989). Also, when the stellar data are corrected for a mysterious temperature dependence, the rise in [N/O] identifying secondary $\mathrm{N}$ nearly disappears (Carbon et al. 1987). Thus $\mathrm{N}$ probably does have a primary contribution, but it can be no larger than in our " $b$ " models. Otherwise $N$ would exceed the requirements that $[\mathrm{N}] \approx 1$ and $[\mathrm{N} / \mathrm{O}] \approx 1$ at $t=8.5 \mathrm{Gyr}$ in $\mathrm{M} 1 \mathrm{~b}$.

Note that the growth of $[\mathrm{N}]$ in M1a scales roughly as $[Z]^{2}$ (Fig. 2). This is because the rate of secondary $\mathrm{N}$ production depends on the initial abundance of $\mathrm{C}$ and $\mathrm{O}$ in stellar envelopes. Since $\mathrm{C}$ and $\mathrm{O}$ scale roughly linearly with $Z$, we have to a good approximation $d N / d Z \propto Z$ and thus $[N] \approx[Z]^{2}$. This relation breaks down for rapidly evolving systems ( $\$ 2.3$ below) because the finite stellar lifetimes delay the enrichment by lower mass stars. In particular, the yield of $\mathrm{N}$ is no longer proportional to the current gas phase $\mathrm{C}$ and $\mathrm{O}$ abundances. The strict equality $[N]=[Z]^{2}$ holds only in the "instantaneous recycling" approximation, where the finite stellar lifetimes are ignored.

The formation of $\mathrm{C}$ in intermediate-mass stars produces a

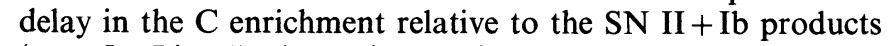
(e.g., O; Fig. 1). At early epochs $\mathrm{C}$ also derives from $\mathrm{SN}$ II + Ib's, but these events account for only $\sim 25 \%$ of the current gas phase $\mathrm{C}$ (at $\sim 13 \mathrm{Gyr}$ in M1a). In M1b the yield and final abundance of $\mathrm{C}$ are slightly lower because some of the newly created $\mathrm{C}$ in intermediate-mass stars is converted to primary. As noted in $\S 2.1$, the early value of $[\mathrm{C} / \mathrm{O}] \sim 0.25$ and the delayed rise to $[\mathrm{C} / \mathrm{O}] \sim 1$ are both within the uncertainties of the observed behavior of C in our Galaxy (Abia et al. 1991; Nissan 1990; Wheeler et al. 1989).

At early epochs the $\mathrm{Fe}$ abundance is controlled by $\mathrm{SN}$ II + Ib's (Fig. 1). The additional Fe enrichment from SN Ia's is delayed by the finite lifetimes of SN Ia precursors $(\S 2.1)$. Because most of the enrichment comes from the more numerous lower mass systems, a "typical" timescale for the Fe rise is $\sim 1-2$ Gyr (Fig. 1). Models M1 predict that $\sim 80 \%$ of the current gas phase $\mathrm{Fe}$ (at $t=13 \mathrm{Gyr}$ ) derives from SN Ia's. This result, and the calculated rise in $[\mathrm{Fe} / \mathrm{O}]$ from $\sim 0.2$ to $\sim 1.0$ beginning at $\sim 1 \mathrm{Gyr}$ (corresponding to $Z \sim 0.1 Z_{\odot}$ or $[\mathrm{Fe} /$ $\mathrm{H}] \sim 0.04)$, are consistent with observations of $\mathrm{Fe}$ in the Galaxy (cf. Abia et al. 1991; Bessel, Sutherland, \& Ruan 1991; and the reviews by Wheeler et al. 1989 and Pagel 1992). However, some estimates of $[\mathrm{Fe} / \mathrm{O}]$ and $[\mathrm{Fe} / \mathrm{Ca}]$ indicate that these ratios have minimum values of $\sim 0.3$ to $\sim 0.5$ at low $Z$ (Nissan 1990). Therefore Fe may be underproduced by $\mathrm{SN}$ II + Ib's in our models by up to a factor of $\sim 2$. Such a change would lower the SN Ia rates we derive by $\sim 25 \%$ (see above).

Hydrogen and $\mathrm{He}$ change relatively little from their primordial values. The growth rate of He relative to $Z$ over the life of the model galaxy is $\Delta Y / \Delta Z \sim 1.1$, where $\Delta Y$ and $\Delta Z$ are the mass fraction gains in He and $Z$, respectively. Recent observational estimates of $\Delta Y / \Delta Z$ range from $\sim 1$ to $\gtrsim 3$ (Peimbert 1986; Pagel, Terlevich, \& Melnick 1986; Pagel 1989; Baldwin et al. 1991; Pagel et al. 1992). There are several possibilities for increasing $\Delta Y / \Delta Z$ in the models if needed (cf. Maeder 1990; Schild \& Maeder 1985). However, these changes would not significantly alter the line ratios in most cases. For example, increasing $\Delta Y / \Delta Z$ to 3 in $M 1$ would only raise the He abundance from $\sim 26 \%$ to $\sim 30 \%$ at $Z=1 Z_{\odot}$. The more important changes for the line ratos occur in the metal abundances, which increase by decades relative to He. Nonetheless, we note that $\Delta Y / \Delta Z=3$ in any model would limit $Z$ to $\lesssim 10 Z_{\odot}$, because of the rapid hydrogen depletion. In our models M2-M6 below, much higher $Z$ 's can occur because $\Delta Y / \Delta Z$ is less than unity.

\subsection{General Abundance Evolution}

All of the remaining models listed in Table 1 use the same nucleosynthesis parameters as the solar neighborhood cases, including the fraction $A \approx 0.1$ of SN Ia precursors. They differ only in the shape of the IMF and/or the timescales for infall and star formation. In $\S 4.1$ below we will show that shorter timescales and IMFs favoring massive stars are required to match the observed $\mathrm{N} \mathrm{V/C} \mathrm{IV} \mathrm{and} \mathrm{N} v / \mathrm{He}$ II line ratios in high-redshift QSOs (also HF92). Here we describe the general trend in the abundance/metallicity evolution in those models.

The metallicity evolution in all six models is plotted Figure 3. The detailed shapes of the curves depend on the functional form of the star formation rate, $\Psi(t)$ (eq. [2]). However, the relative differences between the models, and the "final" metallicities they attain, are not sensitive to the birthrates. For example, if the birthrates followed the square of the density, all of the curves would rise more quickly at younger ages and less quickly later on. But their relative positions in Figure 3 would not change and their "final" metallicities would be very similar to those shown.

The solar neighborhood case M1 has the lowest $Z$ at all epochs. Model M2 uses a flatter IMF ( $x=1$ for all masses) which leads to more processing by high-mass stars and thus higher $Z$ 's. This IMF requires a higher star formation rate, $v$, compared to $\mathrm{M} 1$ in order to preserve the timescale for gas consumption $t(15 \%)=13 \mathrm{Gyr}$ (Table 1$)$. The greater role of high-mass stars is evident in the lower $\mathrm{C} / \mathrm{O}$ and $\mathrm{Fe} / \mathrm{O}$ ratios at late epochs. Also, the secondary $\mathrm{N}$ from high-mass stars dominantes the $\mathrm{N}$ production even when primary $\mathrm{N}$ is included (in the " $b$ " models in Fig. 1).

Model M3 uses a solar neighborhood IMF but shorter evolutionary timescales. The infall time for model M3 (and

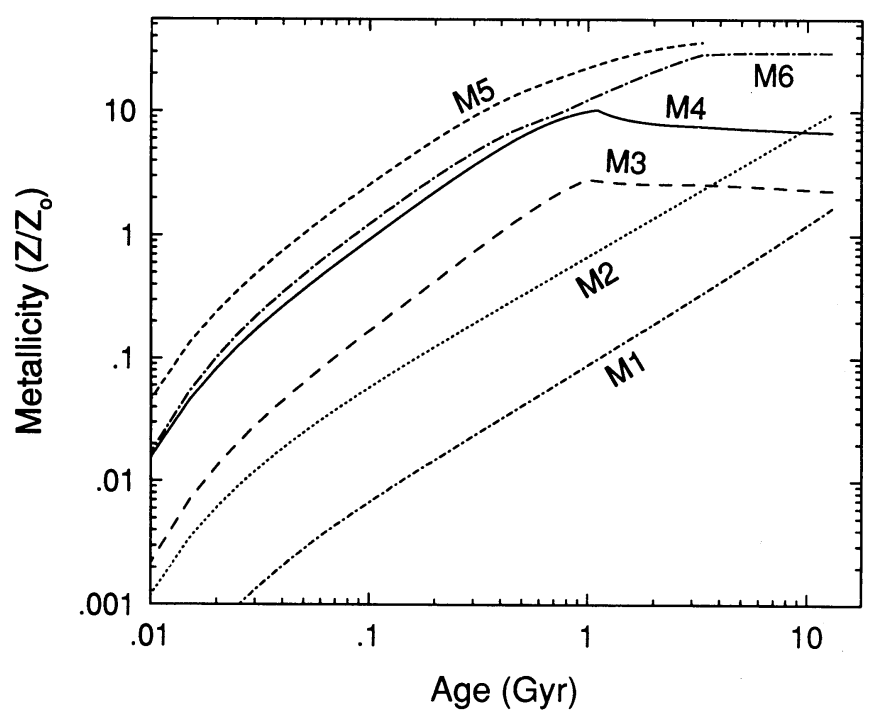

FIG. 3.-Metallicity evolution normalized to solar is plotted for each of the models M1-M6. The solar neighborhood model (M1) achieves $Z=Z_{\odot}$ at the time of the Sun's formation $(\sim 8.5 \mathrm{Gyr})$ but the other models reach substantially higher values of $Z$. 
M4-M6 below) is $0.05 \mathrm{Gyr}$, which is roughly the free-fall time used in models of massive galaxies (Arimoto \& Yoshii 1987; Köppen \& Arimoto 1990). The star formation rate in M3 is almost 20 times larger than $M 1$ so that the $g \approx 15 \%$ mass fraction occurs at $t(15 \%)=0.5 \mathrm{Gyr}$. The net result is that $Z$ rises much more quickly in M3 than M1 or M2. The metallicity reaches a peak at $\sim 1$ Gyr and then declines in M3 (and M4 and M6 below) because the star formation is stopped when the gas fraction reaches $g=3 \%$. After this point $Z$ declines as low-mass stars continue to die and eject lower $Z$ gas into the system. Note that the decline in $Z$ causes the curves in Figure 2 to be double-valued (also for M4). The rapid early star formation and the low gas fractions later on combine to produce large delayed enrichments in both $\mathrm{Fe}$ and $\mathrm{C}$ (Fig. 1). Only $\sim 10 \%$ of the "final" gas phase $\mathrm{Fe}$ is due to SN II + Ib's compared to $\sim 20 \%$ in M1. There is also a small delayed rise in [Mg/O] and [Si/O] (not shown) due to $\mathrm{SN}$ Ia's in models M3-M6. The Fe enrichment is discussed further at the end of this section and in $\S 4.5$.

Model M4 combines a relatively flat IMF $(x=1.1)$ with the short timescales of M3. These parameters are almost identical to the one-zone models of giant elliptical galaxies developed by Arimoto \& Yoshii (1987), Matteucci \& Tornambè (1987), and Angeletti \& Giannone (1990). In $\S 4.1$ we will show that M4 also provides a good fit to many of the high-metallicity-highredshift QSOs. M4 produces considerably higher Z's than models M1-M3, peaking at $\sim 10 Z_{\odot}$ (Fig. 3), and produces a large $\mathbf{N}$ overabundance due to secondary processing in massive stars (Figs. 1 and 2). Note that the secondary $\mathrm{N}$ dominates the $\mathrm{N}$ production in $\mathrm{M} 4 \mathrm{~b}$ even more than in $\mathrm{M} 2 \mathrm{~b}$. The substantial delayed enrichment of $\mathrm{C}$ and $\mathrm{Fe}$ in M4 is less than model M3 because of the flatter IMF (Fig. 1). Also, the ratio $\Delta Y / \Delta Z$ peaks at $\sim 0.5$ in $M 4$, about half the value in $M 1$, because of the lesser emphasis on intermediate-mass stars. The evolution of the main-sequence stellar luminosities and the supernova and star formation rates are described in detail for M4 in $\S 4.5$ below.

Model M5 has slightly faster star formation compared to M4. It also has a flatter IMF $(x=1.0)$ with a lower mass cutoff of $M_{\text {cut }}=2.5 M_{\odot}$. The resulting lack of low-mass stars leads to much less mass locked up in stars and stellar remnants and therefore high gas fractions $g$ for a given $Z$. As a result the metallicities grow very large, exceeding $20 Z_{\odot}$ after $\sim 0.84 \mathrm{Gyr}$. At $20 Z_{\odot}$ the $[\mathrm{N} / \mathrm{C}]$ and $[\mathrm{N} / \mathrm{He}]$ abundances are $\sim 25$ and $\sim 95$, respectively, in both M5a and M6a (below). The system never runs out of gas as in M3 and M4, but the enrichment eventually stops when the interstellar hydrogen is exhausted and the metallicities reach $\sim 35 Z_{\odot}$ (Fig. 2). Obviously, the calculations are uncertain under these conditions because the high $Z$ 's and depleted $\mathrm{H}$ would alter the stellar structure and nucleosynthesis. Nonetheless, truncated IMFs like that used in M5 have been proposed for starburst galaxies (cf. the review by Scalo 1990), and the timescales are still comparable to the models of giant elliptical galaxies. Also, the abundance ratios at early epochs, when $Z \lesssim 10 Z_{\odot}$, evolve almost identically with the giant elliptical model M4 (Figs. 1 and 2). M5 becomes extreme, and possibly unphysical, only in the late stages when the star formation is allowed to run its course.

Model M6 shows that high metallicities and large N/O ratios can also occur with a steep IMF, as in M1, if the IMF is again truncated at $M_{\text {cut }} \sim 2.5 M_{\odot}$. This IMF consumes gas faster than M5, so $g$ eventually reaches $3 \%$ and the star formation is halted before the $\mathrm{H}$ is exhausted. Nonetheless large $Z$ 's are attained, exceeding $[Z]=20 Z_{\odot}$ after $\sim 1.8 \mathrm{Gyr}$. The greater role of intermediate-mass stars is evident in the larger late rise in $\mathrm{C} / \mathrm{O}$ and $\mathrm{Fe} / \mathrm{O}$ and the larger peak value of $\Delta Y$ / $\Delta Z \approx 0.7$ compared to models M4 and M5 (Fig. 1).

Finally, the $\mathrm{Fe}$ overabundances in models M3-M6 are caused by the combined effects of the rapid early star formation, the time delays intrinsic to $\mathrm{SN}$ Ia events, and the relative numbers of SN Ia's and SN II + Ib's caused by the IMF. In general, IMFs favoring low-mass stars produce more SN Ia's and thus larger $\mathrm{Fe}$ enhancements, e.g., [Fe/O]. For example, M3 and M6 have larger Fe enhancements than M4 and M5 because their steep IMFs produce more SN Ia's. The importance of rapid evolution can be seen by comparing $\mathrm{M} 1$ and M3. Both models use a solar neighborhood IMF, but the fast evolution in M3 causes a large disparity in the SN Ia and SN $\mathrm{II}+\mathrm{Ib}$ rates. In particular, in $\mathrm{M} 3$ the $\mathrm{SN}$ Ia rate peaks (at $t \gtrsim 0.5 \mathrm{Gyr})$ then the gas is greatly depleted $(g<10 \%)$ and the star formation and SN II + Ib rates are only about $14 \%$ of their earlier peak values. In fact, in M3, M4, and M6 the star formation and SN II + Ib's have stopped altogether while the SN Ia's are still occurring frequently (see Fig. $9 \mathrm{n} \S$ 4.5). Consequently, the $\mathrm{Fe}$ abundance grows rapidly at late epochs compared to the $\mathrm{SN}$ II + Ib products such as $\mathrm{O}$. The low gas fractions at later times also enhance the impact of each $\mathrm{SN}$ Ia on the abundances. The net result is a rapid rise in $[\mathrm{Fe} / \mathrm{O}]$ from $\sim 0.2$ to $\sim 5$ in M3, to $\sim 2$ in M4 and M5, and to $\sim 10$ in M6 after $\sim$ 1-2 Gyr (Fig. 1).

\section{SPECTRAL SYNTHESIS CALCULATIONS}

Before we apply the evolution models to the observed line ratios, we must be able to predict the line strengths for different chemistries in the broad line gas. We use the photoionization spectral synthesis code CLOUDY (Ferland 1993) with abundances from the enrichment models discussed above. The calculations are made fully self-consistent by feeding the abundances directly into the spectral synthesis code at each time step. In this way we predict the evolution of the line strengths and ratios in each of the enrichment models. We implicitly assume that the QSO emission lines form in wellmixed interstellar gas. The models are intended to apply only to the (inner) high-ionization part of the BLR where the $\mathrm{N} \mathrm{v}$, $\mathrm{He}$ II, and $\mathrm{C}$ IV emission lines form. We are not interested in the lower ionization regions, e.g., of C III] $1909 \AA$, which could well have different physical conditions (Ferland et al. 1992).

The shape of the incident continuum is an important parameter in the photoionization simulations. Observationally, the continuum from typical AGNs is defined better now than ever before. The new observations have brought with them new questions of just how much of the observed continuum is formed within the BLR radius, and just what continuum the BLR clouds actually see (see, for example, Barvainis 1990). For instance, it now seems clear that the continuum longward of 1 $\mu \mathrm{m}$ is formed by grains outside the BLR radius (Sanders et al. 1989). The soft X-ray continuum is probably modified by the “Compton reflector" (Lightman \& White 1988).

We begin with the continuum derived by Mathews \& Ferland (1987, hereafter MF). This continuum was chosen to have the correct X-ray-to-UV continuum power index $\alpha_{o x}$ for radio-quiet objects $(\sim 1.4)$, and an overall spectrum consistent with the observations. We then modified this continuum as follows. We choose an infrared break at $1 \mu \mathrm{m}$, with the continuum slope given by $f_{v} \propto v^{5 / 2}$ for longer wavelengths. This is done to minimize free-free heating, and to be consistent with 
current theories concerning the origin of the near-IR continuum (Sanders et al. 1989).

The big bump component of the MF continuum was originally constrained to reproduce the observed equivalent width of He II $1640 \AA$, but is very poorly constrained in the region between 4 ryd and $500 \mathrm{eV}$. The $\mathrm{C}$ IV/ $\mathrm{N}$ v ratio has little dependence on continuum shape at these energies, since the lines have fairly similar excitation and ionization potentials. However, the $\mathrm{N} \mathrm{v/He}$ II ratio is a ratio of collisional to recombination lines and hence is sensitive to the shape of this continuum because of the resulting temperature dependence (this is discussed extensively in Baldwin et al. 1993). We altered the $\mathrm{X}$-ray continuum to better match the observations of both $\mathrm{N}$ v/He II and $\mathrm{N}$ v/C IV (e.g., Figs. 4-6 below). In particular, we raised the flux at $365 \mathrm{eV}$ by a factor of 4 (see Table 1 of MF) resulting in $\alpha_{o x} \approx 1.24$. Increasing the continuum by this amount had little effect on the $\mathrm{N} v / \mathrm{C}$ IV ratio, but raised the $\mathrm{N}$ v/He II ratio by $\sim 50 \%$. The increase in the MF continuum at these energies is further supported by the fairly common detection of a very soft X-ray excess (Wilkes \& Elvis 1987) and by the fact that the observed X-ray continuum is probably diminished by the Compton reflector. Other than these changes in the IR break and $365 \mathrm{eV}$ flux, the adopted continuum is identical to the MF one. No attempt has been made to change the continuum shape with luminosity (Avni \& Tannanbaum 1986).

To complete the photoionization simulations, we must also specify the pressure law, density, column density, and flux of ionizing photons. We use constant density clouds to avoid the radiative instability problems inherent in constant pressure clouds at these radiation densities (Elitzur \& Ferland 1986). Actually, the choice of pressure law has little influence on the emergent continuum. We make all clouds thick enough to have fully formed the $\mathrm{He}$ II, N v, and $\mathrm{C}$ IV emission lines. Finally, we choose a total hydrogen density of $10^{10} \mathrm{~cm}^{-3}$ and a flux of ionizing photons of $10^{20} \mathrm{~cm}^{-2} \mathrm{~s}^{-1}$ as our fiducial case. This corresponds to an ionization parameter of $\frac{1}{3}$, defined here as the dimensionless ratio of ionizing photon to $\mathrm{H}$ number densities. This pair of density and flux provides a good simultaneous match to the $\mathrm{N} \mathrm{v/He} \mathrm{II} \mathrm{and} \mathrm{N}$ v/C IV observations for the adopted continuum ( $\$ 4.1$ and 4.2 , below). It also nearly maximizes the $\mathrm{N} v / C$ IV and $\mathrm{N} v / H e$ II ratios for a given $Z$, so that the data are "fit" with conservatively low values of $Z$ and the $\mathrm{N} / \mathrm{C}$ and $\mathrm{N} / \mathrm{He}$ abundances. The work of Ferland \& Hamann (1993), Hamann \& Ferland (1993), and Baldwin et al. (1993) shows that the largest $\mathrm{N} \mathrm{v/He} \mathrm{II} \mathrm{and} \mathrm{N}$ V/C IV ratios obtain generally for ionization parameters $\sim \frac{1}{3}$. However, larger fluxes and densities would increase the predicted ratios somewhat. For example, increasing both the flux and density by a decade would raise the line ratios by less than a factor of 2 (Baldwin et al. 1993; Hamann \& Ferland 1993). Some calculations with different parameters are discussed in $\$ \$ 4.1$ and 4.2 below. Detailed discussions of the sensitivity to the flux, density, and continuum shape are given by Baldwin et al. (1993). That work also shows that models with "optimized" continuum shapes or in collisional ionization equilibrium also fail to match many of the observed line ratios with solar abundances.

The densities discussed in this paper apply strictly to the total hydrogen number density in solar metallicity gas. At higher metallicities $\mathrm{H}$ becomes depleted, i.e., the mass fraction $[\mathrm{H}]$ declines, as $Z$ rises. If we had kept the $\mathrm{H}$ density fixed while increasing $Z$, the enhanced metals and the free electrons they provide would have cooled the gas extensively. We therefore scaled the $\mathrm{H}$ density in the models by a factor equal to the depletion of $\mathbf{H}$ relative to solar, i.e., by $[\mathrm{H}]$. This essentially fixes the free electron density and thus preserves the ionization structure of the gas. (In practice the free electron density declines by $\lesssim 20 \%$ between $Z \simeq 1 Z_{\odot}$ and $Z \approx 10 Z_{\odot}$.) The scaling can therefore be thought of as fixing the ionization parameter, if that parameter is redefined as the ratio of ionizing photon to free electron densities.

\section{CHEMICAL EVOLUTION IN QSOS}

\subsection{Comparisons with $\mathrm{N}$ v/C IV and $\mathrm{N}$ v/He II Observations}

Figure 4 compares the predicted and observed $\mathrm{N} v / \mathrm{C}$ IV and $\mathrm{N} v / \mathrm{He}$ II ratios using abundances from $\S 2$ and spectral synthesis parameters from $\S 3$. This figure is similar to Figure 2 in HF92, except that it includes $\mathrm{N}$ V/He II and the model curves are plotted for only one cosmology $\left(H_{0}=75 \mathrm{~km} \mathrm{~s}^{-1} \mathrm{Mpc}^{-1}\right.$ and $q_{0}=\frac{1}{2}$ ). As in HF92, we assume the chemical evolution begins immediately after the big bang. A significant delay
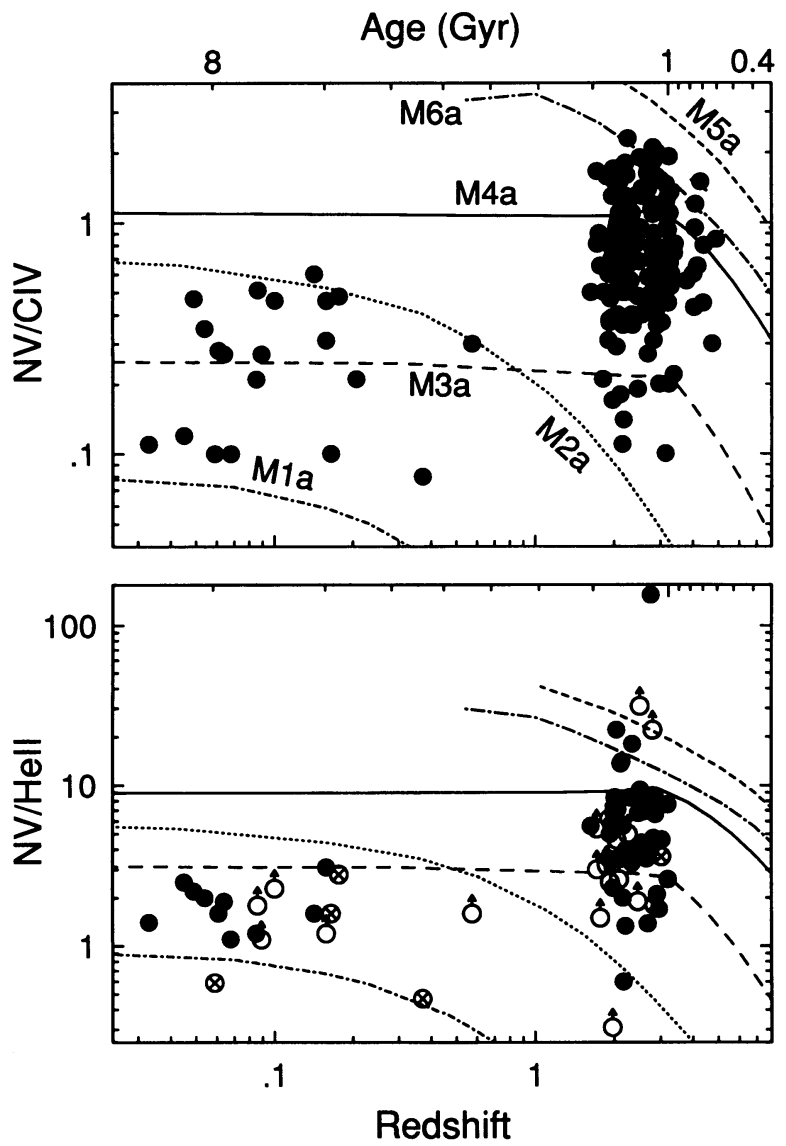

Fig. 4.- - Theoretical $\mathrm{N} v / \mathrm{C}$ IV (top panel) and $\mathrm{N} v / \mathrm{He}$ II (bottom) line ratios for models M1a-M6a are compared to QSO observations for a cosmology with $H_{0}=75 \mathrm{~km} \mathrm{~s}^{-1} \mathrm{Mpc}^{-1}$ and $q_{0}=\frac{1}{2}$. The model curves are plotted against redshift assuming the evolution begins with the big bang. The ages given at the top can be used to identify the model abundances using Figs. 1 and 3. The open circles in the $\mathrm{N}$ v/He II plot are lower limits because $\mathrm{He}$ II is not detected. The crossed circles might be lower limits if the broad feature near He II $1640 \AA$ has some contribution from O III] $1663 \AA$. Note that the solar neighborhood model (M1a) clearly does not match the data. Good fits to most of the high-redshift sources are achieved only if the timescales are shortened and the IMF favors massive stars (e.g., M4a-the giant elliptical model). See $\S 4.1$. 
would shift the model curves to the left. The data points in Figure 1 were measured by us from published spectra in Kinney et al. (1991), Sargent Steidel, \& Boksenberg (1989), and Schneider, Schmidt, \& Gunn (1989a, b, and 1991), or were taken directly from tabulated line strengths in Baldwin et al. (1992), Barthel, Tytler, \& Thomson (1990), Baldwin, Wampler, \& Gaskell (1989), Wilkes (1984, 1986), Uomoto (1984), Young, Sargent, \& Boksenberg (1982), Baldwin \& Netzer (1978), and Osmer \& Smith (1977). For the Barthel et al. data we converted equivalent widths to flux ratios by assuming all of the continua vary as $f_{\lambda} \propto \lambda^{-1.5}$. For the few sources with repeated observations, we used the most recent results. We excluded sources with obvious broad absorption lines because they can diminish the emission line fluxes.

The N V $1240 \AA$ line is usually blended with Ly $\alpha 1216 \AA$ in QSO spectra. We "deblended" these lines by estimating the Ly $\alpha$ wing and attributing the flux above the wing to $\mathrm{N} \mathrm{v}$. When $\mathrm{N} \mathrm{v}$ is weak or severely blended, it is impossible to assign a reliable upper limit to the $\mathrm{N} v$ flux. We therefore recorded $\mathrm{N} \mathrm{v/C} \mathrm{IV} \mathrm{and} \mathrm{N} v / \mathrm{He}$ II ratios only if $\mathrm{N} v$ could be measured directly. None of the sources with redshift $>4$ were excluded for this reason, but there are generally more low line ratio objects than are shown in Figure 4. (Note that the need to deblend $\mathrm{N} v$ introduces a bias against very broad line sources in our sample.) The true $\mathrm{N} v / \mathrm{He}$ II distribution should also contain more large line ratios than appear in Figure 4, because many sources have $\mathrm{N} v$ but no measurable He II. Also He II upper limits were usually not available from the literature and the flux in He II $1640 \AA$ is sometimes blended with O III] 1663 $\AA$. In both our measurements (from the Kinney et al. 1991 spectra only) and those taken from the literature we distinguish between true $3 \sigma$ upper limits on He II, where no measurable line was present, and the cases where a broad line is present but the contribution from $\mathrm{O}$ III] is unknown. In Figures 4-6 the ratios derived from the true upper limits are designated by open circles, and those with a possible $\mathrm{O}$ III] contribution are crossed circles.

The model fits to the $\mathrm{N} v / \mathrm{C}$ IV and $\mathrm{N} v / \mathrm{He}$ II observations reaffirm the conclusions in HF92. In spite of the changes made here to the nucleosynthesis ( $\$ 2.1)$, and the new self-consistent treatment of the abundances $(\S 3)$, the same trends emerge. The solar neighborhood model (M1a) does not fit the data, especially at high redshift, because the timescales are too long and the $\mathrm{N} / \mathrm{C}$ and $\mathrm{N} / \mathrm{He}$ abundances are too low. Including primary $\mathrm{N}$ (M1b-not shown) raises the ratios at higher redshifts, but only to about the same maximum level shown for M1a. Increasing the role of high mass stars by flattening the IMF (model M2a) produces greater $\mathrm{N}$ abundances (and metallicities), but the timescales are still too long to fit the high-redshift data. Shortening the timescales while keeping the solar neighborhood IMF (model M3a) causes [N] and [Z] to rise sooner, but the resulting line ratios are too small and can only match some sources.

The only way to fit the large ratios at high redshift is to both shorten the timescales and use an IMF that favors high-mass stars. The model that fits many of the high-redshift sources (M4a) has a star formation timescale of $t(15 \%) \approx 0.5 \mathrm{Gyr}$ and an IMF slope of $x=1.1$ (Table 1). This is essentially the longest timescale allowed by the data if $q_{0} \approx \frac{1}{2}$. Timescales as long as $\sim \overline{2}$ Gyr could also fit if $q_{0} \approx 0$ (HF92). At $\sim 1 \mathrm{Gyr}$ in M4a the metallicities reach a peak near $\sim 10 Z_{\odot}$ and the $[\mathrm{N} / \mathrm{C}]$ and $[\mathrm{N} / \mathrm{He}]$ abundances are $\sim 10$ and $\sim 38$, respec- tively. Including primary $\mathrm{N}$ in this model (M4b) does not significantly alter the results shown in Figure 4.

The largest line ratios in Figure 4 might require more "extreme" evolution (to $Z \gtrsim 10 Z_{\odot}$ ) as described by models M5a or M6a. However, Baldwin et al. (1993) have shown that the largest $\mathrm{N} v / \mathrm{C}$ IV and $\mathrm{N}$ v/He II ratios (excluding the probably spurious point at $\mathrm{N} v / \mathrm{He}$ II $=155$ ) can be fitted with $Z \sim 10 Z_{\odot}$ abundances if the flux, density, and continuum shape are simultaneously optimized in the spectral synthesis. The optimized parameters would predict $<3$ times larger line ratios for a given $Z$. However, they are also finely "tuned" and there is no reason to believe they apply generally to the QSOs. Additional "tuning" would be required to fit $\mathrm{N} \mathrm{v} / \mathrm{He}$ II and $\mathrm{N}$ v/C IV simultaneously at each $Z$ (as we have tried to do here; see $\$ \S 3$ and 4.2, below) because the conditions that maximize $\mathrm{N} \mathrm{v} / \mathrm{He}$ II do not necessarily maximize $\mathrm{N}$ v/C IV. In any case, even the optimized parameters require enhanced $[\mathrm{N} / \mathrm{He}]$ and $[\mathrm{N} / \mathrm{C}]$ abundances in many high redshift sources (Ferland \& Hamann 1993; Hamann \& Ferland 1993; Baldwin et al. 1993). Without making severe and probably unphysical changes to the stellar yields, the only way to meet this requirement is to use an IMF that favors high-mass stars - or at least excludes the low-mass ones-and produces high metallicities $(\S 2.3)$.

\subsection{The $\mathrm{N} \mathrm{v} / \mathrm{He}$ II versus $\mathrm{N} \mathrm{v} / \mathrm{C}$ IV Correlation}

Figure 5 shows that the observed $\mathrm{N}$ v/He II and $\mathrm{N}$ v/C IV line ratios are correlated and well predicted by the models if the fiducial physical parameters from $\S 3$ are used. The data are the same as in Figure 4 and the theoretical curves represent models M1a-M6a. The metallicities labeled on the curves strictly apply for M5a, but they are also approximately correct for M2a, M4a, and M6a where they overlap (see Fig. 2). The good fit to the data using a one-zone photoionization model with fixed parameters suggests that the observed trend results largely from abundance/evolution differences. The scatter can be explained by different physical conditions in each object. It is worth emphasizing that changes in the physical conditions alone for any fixed $Z \lesssim Z_{\odot}$, cannot account for the full range

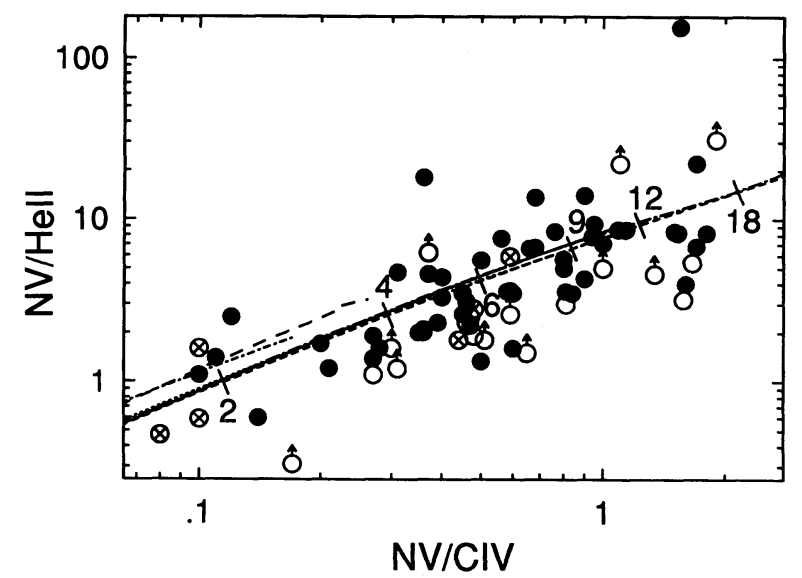

Fig. 5.-Observed $\mathrm{N} v / \mathrm{C}$ IV and $\mathrm{N} v / \mathrm{He}$ II line ratios are shown to be weakly correlated with each other and well predicted by the models. The data symbols and the model lines are the same as in Fig. 4. The tick marks labeled 2, $4,6,9,12$, and 18 indicate the metallicity $[Z]$ in model M5a (short dashed line). These $[(Z)$ labels are also approximately correct for models M2a, M4a, and M6a across the metallicity ranges they encompass. See Fig. 3 and $\S 4.2$. 
of observed ratios. It is possible to match the range of observations by varying the physical parameters in a $Z \sim 10 Z_{\odot}$ model (Hamann \& Ferland 1993; Ferland \& Hamann 1993; Baldwin et al 1993). However, simple changes in the physical conditions would not follow the overall slope in Figure 5 without fine tuning several parameters at once. Different physical conditions are therefore likely to contribute more to the scatter in Figure 5 than to the correlation.

The effects of varying the flux, density, and $\alpha_{o x}$ are illustrated in Figure 6. All of the curves in this figure use the same abundance model, M5a. The solid line in the center panel marks the "standard" parameter case (§3) and is identical to the M5a curve in Figure 5. The good fit of this line to the data motivated our original choice of parameters in $\S 3$. In a later paper we will consider how systematic trends in the continuum



FIG. 6.-Observed $\mathrm{N} \mathrm{v/C} \mathrm{IV} \mathrm{and} \mathrm{N} v / \mathrm{He}$ II line ratios are compared to the abundance model M5a for different values of the density, photon flux, and $\alpha_{o x}$ used in the spectral synthesis. Different values of $\alpha_{o x}$ are used in each panel, as labeled in the lower right. The combinations of density and ionizing photon fluxes are indicated in the top panel. For example, 10, 20 indicates a density of $10^{10} \mathrm{~cm}^{-3}$ and a flux of $10^{20} \mathrm{~cm}^{-2} \mathrm{~s}^{-1}$. The solid line in the center panel uses the fiducial parameters as shown for M5a in Fig. 5. The data symbols are the same as in Fig. 4. See also $§ 4.2$. shape, that might be correlated with abundance (see $\S 4.3$ below), can influence the fits in Figure 6.

\subsection{Evidence for a Mass-Metallicity-Redshift Relation}

The observed trend for larger $\mathrm{N}$ v/C IV and $\mathrm{N}$ v/He II line ratios at high redshift in Figure 4 is not due to measurement errors. However, the low-redshift data were measured exclusively by us (from the Kinney et al. 1991 spectra) while the high-redshift results derive from various references $(\S 4.1)$. We therefore consider the possibility of systematic errors in the measurements. First, we not that among the high-redshift data there are no substantial differences between the various data sets. Second, to understand the uncertainties in our lowredshift measurements we each (the authors) measured the lines from Kinney et al. (1991) and compared our results. One set of measured ratios was systematically higher - typically by $\sim 20 \%-30 \%$ but as much as a factor of $\sim 2$-because the deblending of $\mathrm{N} v$ from Ly $\alpha$ is largely subjective. We adopted the measurement set with the largest line ratios to minimize the difference between the high- and low-redshift data. Nonetheless, the largest ratios at low redshift are $\lesssim \frac{1}{3}$ of the largest high-redshift ratios.

This trend was first noted by HF92 for N v/C IV only. Systematic changes in the continuum shape and physical conditions with redshift could contribute $(\S 4.2)$. However, the different $\mathrm{N} / \mathrm{C}$ and $\mathrm{N} / \mathrm{He}$ abundances predicted by the enrichment models, with fixed spectral synthesis parameters, provide a neutral explanation for the trends in Figures 4-6. Thus the models predict metallicities that are typically several times higher at high redshift. If the high-redshift QSOs evolve into lower redshift QSOs and AGNs, the trend in the line ratios with $z$ suggests declining metallicities and $\mathrm{N} / \mathrm{C}$ and $\mathrm{N} / \mathrm{He}$ ratios as the systems age. This would require an influx of lowmetallicity gas, such as by galaxy mergers or by the continuing infall from a galactic disk or halo. Alternatively, if the highredshift QSOs are short lived and unrelated to the low-redshift systems, the models imply that the most extensive chemical evolution occurs only at early cosmological epochs.

In nearby elliptical galaxies there is a well-known trend for higher metallicities in more massive systems (cf. Tinsley 1980; Pagel \& Edmunds 1981; Vader 1986a; Bica 1988). This correlation is believed to be due to the action of galactic winds driven by supernovae (Arimoto \& Yoshii 1987; Matteucci \& Tornambè 1987; Angeletti \& Giannone 1990). The massive ellipticals are more tightly bound and thus better able to retain their gas while they evolve toward high $Z$. Less massive systems lose their gas before high metallicities are attained. By analogy with the ellipticals, the highest metallicity QSOs might also be the most massive. In this case a metallicity-redshift correlation would suggest that the most massive QSOs (and/or host galaxies) form preferentially at early epochs. Studies of elliptical galaxies suggest that the highest $Z$ 's we infer at highredshift require the deep gravitational well of systems with total mass $\sim 10^{12} M_{\odot}$ (also Bica et al. 1988).

Some support for a mass-metallicity-redshift relation in QSOs can be found in the correlation between the line ratios and the luminosities. We derive approximate luminosities, $v L_{v}$ at $\sim 1450 \AA$, for as many of the sources plotted in Figure 4 as possible. For some of the uncalibrated data, e.g., of Barthel et al. (1990), we use continuum fluxes from the other references when available $(\S 4.1)$. We deduce distances from the redshifts assuming $H_{0}=75 \mathrm{~km} \mathrm{~s}^{-1} \mathrm{Mpc}^{-1}$ and $q_{0}=\frac{1}{2}$. We do not 
attempt to correct for Galactic extinction, although the resulting changes should be negligible for sources at high Galactic latitude (Burstein \& Heiles 1978). The results are plotted in Figure 7. There is a weak but clear tendency for larger $\mathrm{N} \mathrm{v/C} \mathrm{IV} \mathrm{and} \mathrm{N} v / \mathrm{He}$ II ratios in more luminous sources. A trend consistent with Figure 7 is also evident in the compilation of low-redshift AGN spectra by Véron-Cetty, Véron, \& Tarenghi (1983). In particular, a quick inspection of their composite spectra grouped by luminosity (their Fig. 5) reveals that sources with $v L_{v} \lesssim 5 \times 10^{9} L_{\odot}$ typically have at least $\sim 5$ times lower $\mathrm{N} v / \mathrm{C}$ IV and $\mathrm{N} v / \mathrm{He}$ II ratios than those with $v L_{v} \gtrsim 5 \times 10^{10} L_{\odot}$. Furthermore, the ratios in Véron-Cetty et al. (1983), although not measured by them, appear to represent an extension of the correlations in our Figures 4-7 to lower redshifts and luminosities. A well-known example of a lowluminosity AGN with small line ratios is NGC 5548 (Clavel et al. 1991), for which we estimate roughly solar abundances (Hamann \& Ferland 1993b).

These trends are naturally explained if there is a correlation between the source luminosity and metallicity. This correlation can, in turn, be interpreted in terms of a mass-metallicity relation if the luminosity is related to the mass of the system and the depth of the galactic potential. Recent work suggests that the luminosities of QSOs and AGNs are indeed well correlated with at least the mass interior to the broad-line regions (Padovani \& Rafanelli 1988; Padovani, Burg, \& Edelson 1990; Koratkar \& Gaskell 1991a). Therefore, the line ratios might be
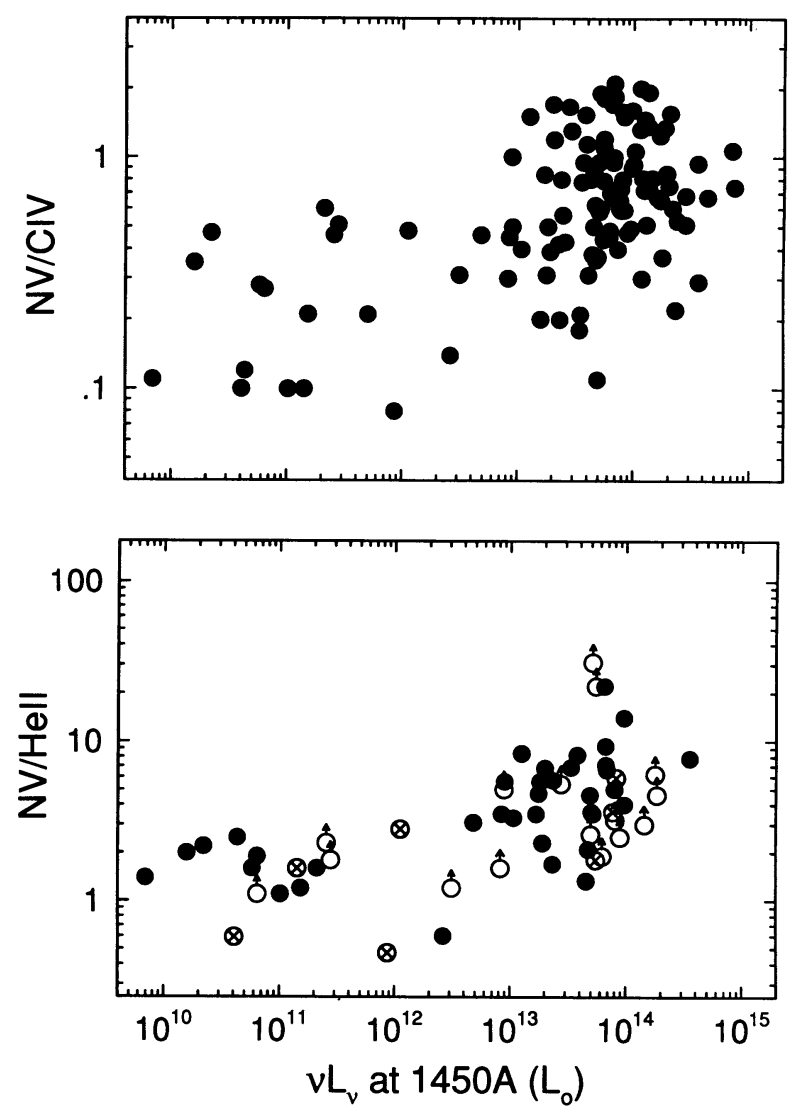

Fig. 7.-Observed $\mathrm{N} v / \mathrm{C}$ IV and $\mathrm{N}$ v/He II line ratios are shown to be weakly correlated with the QSO luminosities. The data symbols are the same as in Fig. 4. See $\S 4.3$. indicators of not only the chemical enrichment, but also the total mass that binds the gas.

The luminosity-metallicity correlation also has implications for the well-known "global Baldwin effect" observed in AGNs and QSOs (Baldwin 1977; Kinney, Rivolo, \& Koratkar 1990). This "effect" describes a relationship between the source luminosity and the $C$ IV equivalent width, in the sense that the $C$ IV line is weaker at larger luminosities. Several factors could contribute to this, but the metallicity correlation implied by Figure 7 is yet another. We are now pursuing this possibility further. Our preliminary results suggest that, even for fixed photoionization conditions, The $\mathrm{C}$ IV equivalent widths do indeed decline with increasing $Z$.

Note that care must be taken in interpreting the line ratioluminosity correlation across such a wide range in redshift. Magnitude-limited samples will prefer luminous sources at higher redshifts, possibly leading to false luminosity correlations (see for example Cheng, Gaskell, \& Koratkar 1991). With J. Baldwin and J. Shields, we are now involved in a program to quantify and distinguish between the trends with luminosity and redshift. This work will use expanded samples at low redshift, include a range of source luminosities in several redshift bins, and consider the influence of systematic trends in the continuum shape for relating the observed line ratios to $Z$.

\subsection{Galactic Winds: Constraints on the Masses and Radii}

Even if the mass-metallicity relation in QSOs is not regulated by galactic winds, or if there turns out to be no massmetallicity relation at all, it is clear that the QSO environments must be bound tightly enough to retain their gas while the chemical enrichment occurs. Galactic winds will "turn on," and presumably halt the enrichment, when the thermal energy input by novae, supernovae, stellar winds, etc., exceeds the gravitational binding energy of the gas. For a given evolution model, the minimum binding energy needed to reach high $Z$ 's leads to an estimate of the minimum mass of the system. In this section we derive the thermal energies from supernovae in models M4-M6 to place lower limits on the masses that bind the QSO star-forming regions.

The thermal energy deposited per supernova $\epsilon(t)$ is a function of the kinetic energy of the explosion and the density of the surrounding gas (Cox 1972; Arimoto \& Yoshii 1987). The total thermal energy of the system at any time $t$ is the integral over all previous times of $\epsilon(t)$ times the supernova rates. We calculate the rates of SN Ia's and SN II + Ib's separately (after Matteucci \& Greggio 1986), but assume that all SN events have the kinetic energy of $10^{51}$ ergs. We therefore add the rates together when deriving the thermal energies. This is mostly a formality because the SN II + Ib's dominate the total $\mathrm{SN}$ rates by factors of at least several in the models.

The total thermal energy of the gas is

$$
E_{\mathrm{th}}(t)=M_{T} \int_{0}^{t} \epsilon\left(t^{\prime}\right) R_{\mathrm{SN}}\left(t-t^{\prime}\right) d t^{\prime},
$$

where $M_{T}$ is the total mass in gas, stars, and stellar remnants, and $R_{\mathrm{SN}}\left(t-t^{\prime}\right)$ is the combined supernova rate per unit mass at time $t-t^{\prime}$ (see also Matteucci \& Tornambè 1987 and Angeletti $\&$ Giannone 1990). Clearly the calculation of $E_{\mathrm{th}}(t)$ is uncertain. For example, in a given model the $\mathrm{SN}$ rates depend critically on the lower mass limit of stars producing SN II + Ib's. Arimoto \& Yoshii (1987) used a very low value of $3 \mathrm{M}_{\odot}$ compared to the $7 M_{\odot}$ adopted here $(\S 2.1)$. Raising the mass cutoff 
from 3 to $7 M_{\odot}$ lowers the SN II + Ib rates by factors of $\sim 4$ to $\sim 10$ for IMF slopes between 1.0 and 1.6, respectively. The supernova rates are also sensitive to the stellar lifetimes, particularly in the short-timescale models M3-M6. For example, the approximate lifetimes adopted by Arimoto \& Yoshii (1987) are larger than Scalo's (1986) by factors of $\sim 4$ at $8 M_{\odot}$ and $\sim 3$ at $18 M_{\odot}$. Using the longer lifetimes in M4 would lead to SN rates which are $\sim 2$ to $\sim 3$ times smaller and thermal energies for $t \lesssim 0.4 \mathrm{Gyr}$. Another uncertainty is the efficiency of radiative cooling of the supernova remnants. Our treatment (from Cox. 1972 as expressed by Arimoto \& Yoshii 1987) does not consider metallicity effects, but high $Z$ 's could enhance the radiative cooling and thus lower the thermal energy. The amount of radiative cooling is also sensitive to the unknown density of the gas into which the $\mathrm{SN}$ remnant expands (see below). Finally, the coupling between the hot expanding remnants and the cooler ambient gas is uncertain. Galactic wind calculations generally assume that the gas is thermally mixed, but in actual galaxies the expanding remnants could blow out a hot gas component while some of the cooler denser gas remains behind.

In spite of these uncertainties we proceed, mainly to show that standard wind calculations do not place strong constraints on the galaxy masses needed to reach large $Z$ 's in QSOs. If the gas and stars are uniformly distributed in a spherical volume, and the system is in virial equilibrium, the gravitational binding energy of the gas is

$$
\Omega(t)=\frac{3 G M_{T}^{2}}{5 R} g(2-g),
$$

where $R$ is the radius, $g$ is the ratio of mass in gas to total mass $(\S 2.2)$, and $G$ is the gravitational constant. The time dependence results from the decline in $g$ as the system evolves. Strictly speaking, $M_{T}$ in equation (4) is the total gravitational mass, which could exceed the mass in gas and stars in equation (3) if dark matter is present. Ignoring the possible dark matter, we use the ratio of equations (3) and (4) to derive the minimum total mass needed to bind the gas and reach a given $Z$ as a function of the system radius. Figure 8 plots the average minimum mass needed to reach $Z \approx 10 Z_{\odot}$ in models M4-M6. The individual models deviate from the plotted average by less than $35 \%$. The solid lines show the average model results for gas densities surrounding the supernovae of $n_{0}=1,100$, and $10^{4} \mathrm{~cm}^{-3}$. In general, the thermal energy and thus the minimum mass scale as $n_{0}^{-0.328}$ (Arimoto \& Yoshii 1987). Masses above these lines are "allowed" in the sense that they have enough binding energy to reach $Z=10 Z_{\odot}$ before the gas is expelled. For example, if $n_{0}=100 \mathrm{~cm}^{-3}$ and the radius of the system is $\sim 1000 \mathrm{pc}$, then $\sim 10^{9} M_{\odot}$ is needed to bind the gas. Less massive systems would lose their gas before $Z \approx 10$ $Z_{\odot}$ is attained. The error bar at the left in Figure 8 shows the approximate shifts required for different choices of $Z$. The dotted lines in the figure show the loci of constant mean total densities, $\bar{n}$. If the gas densities surrounding the supernovae are greater than or equal to the mean total density (i.e., $n_{0} \gtrsim \bar{n}$ ), then the "allowed" regions in Figure 8 are confined to the wedge-shaped section in the upper right bordered by the lines of constant $n_{0}=\bar{n}$.

\subsection{Luminosity Evolution of the Stellar Population}

In this section we estimate the evolution of the stellar UV and bolometric luminosities in the QSO/giant elliptical model M4. For simplicity we assume the stars emit like blackbodies

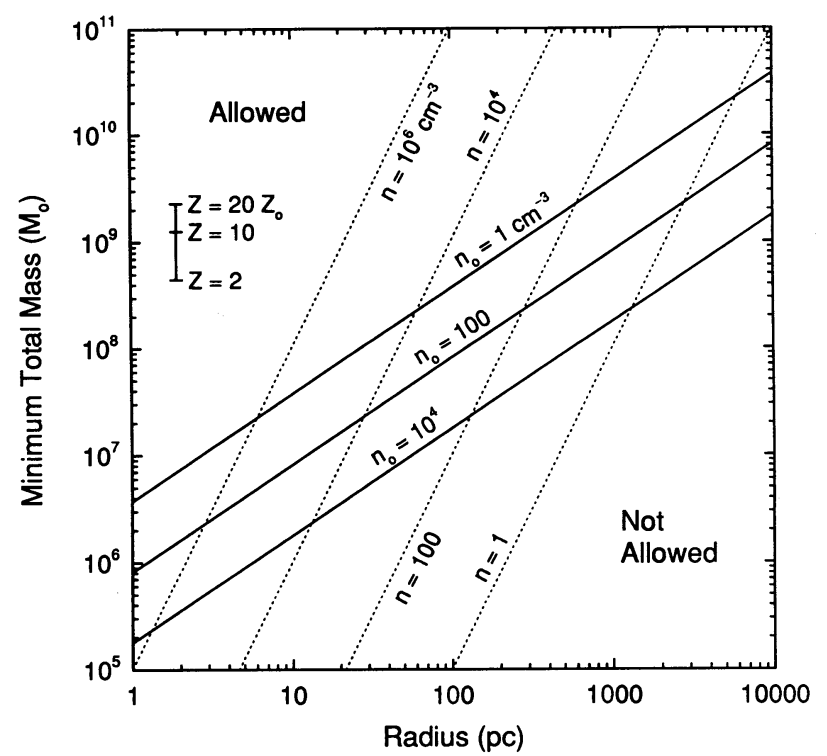

Fig. 8.-Minimum total masses required to reach metallicities of $Z \sim 10$ $Z_{\odot}$ are plotted as a function of the system radius for an average of the QSO models M4-M6. The models are represented by a single curve for each of three gas densities $n_{0}$. Systems with total masses below these curves are "not allowed " because they will lose their gas in a galactic wind before $Z \sim 10 Z Z_{\odot}$ is achieved. See $\S 4.4$. The error bar at the left shows how the location of the curves depends on the choice of $Z$. The densities $n_{0}$ apply to the environment into which the supernova remnants expand. The dotted lines show various mean densities of the entire system, i.e., in gas, stars and stellar remnants.

and consider only stars on the main sequence. These approximations do not significantly affect the UV luminosity because, as long as there is ongoing star formation, the high mass on the main sequence will dominate the UV flux and their spectra are close to blackbodies at the wavelengths of interest $(\sim 1450 \AA)$. The bolometric luminosity is more sensitive to the neglect of post-main-sequence phases because cool supergiants can contribute at later epochs. However, on the short timescales of interest here, only high-mass stars will evolve off the main sequence. They evolve at nearly constant luminosity and, even though they are short lived on the main sequence, their postmain-sequence lifetimes are even shorter (Iben 1967). Therefore, with ongoing star formation, the high-mass population on the main sequence will outnumber and outshine their postmain-sequence counterparts. Thus the high-mass mainsequence stars will also dominate the bolometric luminosity for ages $\lesssim 1$ Gyr.

We calculate bolometric luminosities for the main-sequence stars assuming a stellar mass-luminosity relation of the form $L_{*}=M_{*}^{\alpha}$, with $\alpha=2.7$ for $M_{*}<1 M_{\odot}$ and $\alpha=3.4$ for $M_{*} \geq$ $1 M_{\odot}$. This relation approximates the empirical results of Scalo (1986). We estimate "UV luminosities," i.e., $v L_{v}$ at 1450 $\AA$, by first deriving stellar temperatures from the bolometric luminosities $L_{*}$ and the stellar radii $R_{*}$ in the blackbody formula, $L_{*}=4 \pi R_{*}^{2} \sigma T_{*}^{4}$. The radii are interpolated from Thompson (1984) for $M_{*}^{*}>1 M_{\odot}$ and Allen (1976) for $M_{*}<1$ $M_{\odot}$. This procedure yields a consistent set of blackbody parameters from which we derive $v L_{v}$ using the Planck equation.

The top panel of Figure 9 shows the bolometric luminosities contributed by stars of various masses (thin solid lines), as well as the total bolometric luminosity (thick solid line) and the total UV luminosity ( $v L_{v}$; dotted line). The lower panel shows the 




FIG. 9.-Top panel: luminosity evolution of the main-sequence stellar population in the QSO/giant elliptical model M4. The thin solid lines are the bolometric luminosities contributed by stars in the mass ranges shown. The thick solid line shows the total bolometric luminosity of the whole (mainsequence) population. The dotted line is the total "UV luminosity," i.e., $v L_{v}$ at $1450 \AA$, derived assuming the stars emit like blackbodies at this wavelength. Bottom panel: Star formation (thick line) and supernovae (thin) rates for model M4. The scales at the left applies for the star formation rates, while the right applies for both the SN Ia and SN II + Ib rates. The star formation stops at $\sim 1$ Gyr when the gas mass fraction drops below 3\%. The high-mass stars dominate the total luminosities before the star formation stops. Soon afterward the high-mass stars have all died and the lower mass stars control the luminosities and continue to produce SN Ia's. See $\$ 2.3$ and 4.5 .

corresponding rates of star formation (thick line) and supernova events (thin lines) in the same model. The rates and luminosities scale linearly with the mass of the system. All are plotted for a total evolving mass $M_{T}=10^{11} M_{\odot}$. The peak luminosities coincide with the peak in the star formation rate at $\sim 0.1 \mathrm{Gyr}$. The peak bolometric luminosity scales roughly as $\sim 3 \times 10^{13}\left(M_{T} / 10^{11} M_{\odot}\right) L_{\odot}$. The UV luminosity is $\sim 2$ to $\sim 3$ times less than bolometric because, even though $1450 \AA$ is close to the peak in $L_{v}$, the expression $v L_{v}$ underestimates the full width of the stellar spectra. The figure shows clearly that the hottest, most massive stars dominate the luminosity as long as there is ongoing star formation. Their spectra peak at roughly $1000 \AA$ with nearly a factor of $\sim 2$ drop at the Lyman edge912 A (Kurucz 1979; Leitherer, Gruenwald, \& Schmutz 1992).

\subsection{Delayed Fe Enrichment as a Test of $q_{0}$}

The $\sim 1 \mathrm{Gyr}$ delay in the $\mathrm{Fe}$ enrichment depends on the lifetimes of SN Ia precursors ( $\$ \$ 2.2$ and 2.3) and is not sensitive to the IMF or the timescales used in the models. Therefore, in as much we understand the evolution of SN Ia precursors, the $\mathrm{Fe}$ abundance can be used as a "clock" to constrain QSO ages. When applied to sources at high redshifts, the Fe clock could provide an indirect test of the cosmology. Figure 10 shows explicitly how the redshifts are related to the maximum QSO ages, i.e., the time since the big bang. Observations of $\mathrm{Fe}$ abundances above the nominal SN II + Ib ratios (e.g., $[\mathrm{Fe} / \mathrm{O}] \gtrsim 0.5$ ) would imply that SN Ia's are involved and the QSOs are older than $\sim 1$ Gyr (Fig. 1). At redshift $\sim 5$, ages $\geq 1$ Gyr would require $q_{0}<\frac{1}{2}$ for any $H_{0}>45 \mathrm{~km} \mathrm{~s}^{-1} \mathrm{Mpc}^{-1}$ (Fig. 10). Thus, high $\mathrm{Fe}$ abundances at these redshifts would suggest that the mean density in the universe is less than critical. The converse is not necessarily true, however. Low $\mathrm{Fe}$ abundances do not require ages $\$ 1$ Gyr because the contribution of SN Ia's might be limited by other factors. For example we can imagine extreme IMFs with high lower mass cutoffs that virtually eliminate SN Ia's altogether. Nonetheless, if the IMF is not so greatly skewed toward massive stars, low $\mathrm{Fe}$ abundance in the broad line gas would imply young ages.

This reasoning has already been applied to observations of local galaxies. For example, Worthey, Faber, \& Gonzalez (1992) note that low values of $[\mathrm{Fe} / \mathrm{Mg}]$ in the metal-rich stars of giant ellipticals suggest short evolution timescales. The same conclusion has been drawn from measurements of low $[\mathrm{Fe} / \mathrm{O}]$ in metal-rich Galactic bulge stars (Matteucci \& Brocato 1990; Barbuy \& Grenon 1990). The observations of high $Z$ but low $[\mathrm{Fe} / \mathrm{O}]$ and $[\mathrm{Fe} / \mathrm{Mg}]$ imply substantial enrichment in less than $\sim 1$ Gyr. Analogous observations of low $[\mathrm{Fe} / \mathrm{O}]$ in metal-poor Galactic halo stars suggest that the halo formed in $\$ 1 \mathrm{Gyr}$ (Truran 1987; Wyse \& Gilmore 1988; but see also Smecker \& Wyse 1991).

The validity of $\mathrm{Fe}$ as a clock depends on the identification of long-lived SN Ia systems as a major source of Fe enrichment. Edmunds et al. (1991) and Worthey et al. (1992) and pointed out that if SN II + Ib's of different masses produce different

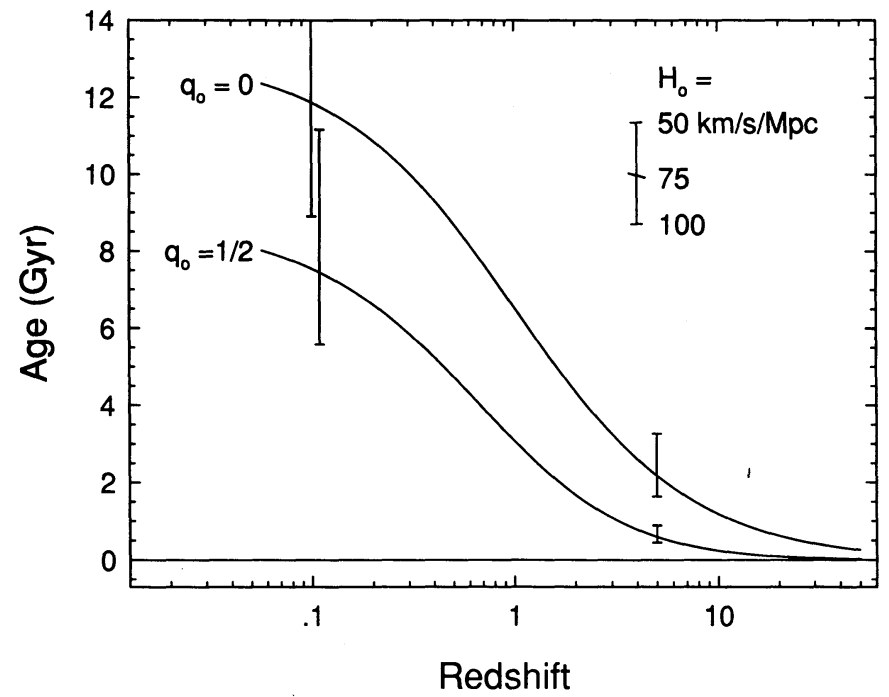

Fig. 10.-Redshift vs. age of the universe is plotted for cosmologies with $H_{0}=75 \mathrm{~km} \mathrm{~s}^{-1} \mathrm{Mpc}^{-1}$ and $q_{0}=0$ and $\frac{1}{2}$. The "error" bars at redshifts 0.1 and 5 show the range of variation possible for $H_{0}$ between 50 and $100 \mathrm{~km} \mathrm{~s}^{-1}$ $\mathrm{Mpc}^{-1}$. The figure shows that ages estimates for high-redshift QSOs can be used to constrain the value of $q_{0}$ (see $\left.\S 4.6\right)$. 
ratios of $\mathrm{Fe} / \mathrm{O}, \mathrm{Fe} / \mathrm{Mg}$, etc., as the nucleosynthesis results suggest (e.g., Arnett 1991; Woosley \& Weaver 1992), then the observed trends for increasing $\mathrm{Fe} / \mathrm{O}, \mathrm{Fe} / \mathrm{Mg}$, etc., with $\mathrm{Fe} / \mathrm{H}$ might be produced by changes in the distribution of exploding core masses with metallicity. The sense of the changes would have to be for fewer high-mass cores at high metallicities and/or later epochs. Such variations with metallicity might be caused, for example, by larger mass loss rates and thus smaller "final" core masses at higher $Z$ (Edmunds et al. 1991). In this scenario SN Ia's might play a negligible role in the Fe enrichment and there would be no connection to a particular timescale. However, we point out that this scenario is ad hoc and may conflict with recent estimates of the rates and yields of $\mathrm{SN}$ Ia's in the Galaxy. In our solar neighborhood models, the yields from SN II + Ib's and SN Ia's and the expected lifetimes of SN Ia precursors, combine to give good agreement with both the $\mathrm{SN}$ rates and the $\mathrm{Fe}$ abundance trends $(\$ 2.2)$.

One-zone photoionization models indicate that the ensemble flux in the UV Fe II lines is sensitive to the $\mathrm{Fe}$ abundance (this work; Wills, Netzer, \& Wills 1985). If the gas is in photoionization equilibrium and the physical conditions are the same in a sample of objects, relative $\mathrm{Fe}$ abundance differences will be discernible. The ratio Fe II (UV)/Mg II (2798 $\AA$ ) offers some promise as an abundance indicator because the ions have similar ionization energies and the ratio $\mathrm{Fe} / \mathrm{Mg}$ is predicted to increase by factors $>3$ (Fig. 1).

One complication for measuring $\mathrm{Fe}$ abundances from $\mathrm{Fe}$ II is that $\mathrm{Fe}$ might be depleted by dust grains in the cool $\mathrm{Fe}^{+}$ environment (Gaskell et al. 1981). Another is that the Fe-rich gas in SN Ia remnants might not cool enough to appear as observable $\mathrm{Fe}$ II in the broad-line regions, or might be ejected from the Galaxy altogether. The predicted Fe overabundances occur when the gas content is low, i.e., $g<10 \%(\S 2.3)$, allowing easy ejection of the hot remnants via a supernova-driven wind (§ 4.4). If ejection does occur, the high $Z$ and $[\mathrm{Fe} / \mathrm{O}]$ gas would enrich the intergalactic medium (also Vader $1986 \mathrm{~b}$ and 1987).

\subsection{Delayed Fe Enrichment and the Strength of $\mathrm{Fe}$ II Emission}

Models M3-M6 also predict that Fe becomes overabundant after $\sim 1-2$ Gyr due to the SN Ia contribution ( $\$ 2.3$ and Figs. 1 and 2). These overabundances, relative to solar ratios, might explain the well-known strong Fe II emission from QSOs and AGNs (cf. Osterbrock 1977; Phillips 1978; Wills et al. 1985). The total flux from Fe II sometimes rivals that of all other species combined. Photoionization/spectral synthesis models that use solar abundances have so far failed by factors of $\sim 2$ to $\sim 10$ to account for the strength of the Fe II emission (Netzer \& Wills 1983; Wills et al. 1985; Netzer 1985; Collin-Souffrin, Hameury, \& Joly 1988). Of particular note is the failure to match the observed Fe II (UV)/Mg II (2798 $\AA$ ) ratio. Wills et al. (1985) note that this requires either an overabundance of $\mathrm{Fe}$ or some selective excitation of Fe II not yet included in the models. We are presently pursuing the possibility that the Fe II flux is enhanced by resonant absorption of Ly $\alpha$ (Penston 1987). Our preliminary results show that Ly $\alpha$ pumping can play a significant role (Hamann, Ferland, \& Johansson 1993). However, the $\mathrm{Fe}$ overabundances of $[\mathrm{Fe} / \mathrm{O}] \sim 2$ to 10 predicted here (Fig. 1) are probably sufficient by themselves to match the observations. If we express the overabundance in terms of $[\mathrm{Fe} / \mathrm{Mg}]$, models M3 and M6 predict close to the mean value of $[\mathrm{Fe} / \mathrm{Mg}] \sim 3$ required to fit the data (Wills et al. 1985).

\section{SUMMARY AND DISCUSSION}

We have combined chemical evolution and spectral synthesis models to interpret the $\mathrm{N}$ V/C IV and $\mathrm{N} v / \mathrm{He}$ II broad emission line ratios of QSOs. Our main results are the following.

1. The line ratios usually imply large $\mathrm{N} / \mathrm{C}$ and $\mathrm{N} / \mathrm{He}$ abundances, from $\sim 1$ to $>10$ times solar. This requires chemically enriched gas with metallicities from $\sim 1$ to perhaps $\gtrsim 10 Z_{\odot}$. IMFs favoring high-mass stars compared to the solar neighborhood are also required for at least the high- $Z$ sources. To match the high-redshift data, the evolution/star formation must occur in $\lesssim 1.0$ Gyr if $q_{0} \approx \frac{1}{2}$, or $\lesssim 2$ Gyr if $q_{0} \approx 0$.

2 . Redshift $\gtrsim 2$ QSOs often have higher metallicities than lower redshift sources. It is difficult to quantify this result because the line ratios used to derive the metallicity are also influenced by the physical conditions in the gas. Nonetheless, metallicity differences do exist, and they provide a natural explanation for the trend with redshift. We suggest that there is a mass-metallicity-redshift relation in QSOs analogous to the well-known relationship in nearby ellipticals. Thus, the highmass QSOs (and/or host galaxies) form preferentially at high redshifts. Some support for a mass-metallicity-redshift relation comes from the positive correlation between the line ratios (i.e., the metallicities) and the source luminosities (possible tracers of the mass). The analogy with the ellipticals suggests that the highest metallicity QSOs reside in galaxies of mass $\sim 10^{12}$ or more $(\$ 4.3)$. The trend in the line ratios with luminosity also suggests that metallicity differences can play a role in the "global Baldwin effect."

3. The delayed enrichment of Fe by SN Ia's can be used as a "clock" to constrain QSO ages and perhaps the cosmology if accurate abundances are measured. The $\sim 1$ Gyr timescales of this clock depends only on the (albeit uncertain) lifetimes of SN Ia precursors and not on the evolution timescales used in the models. One-zone photoionization models suggest that the ratio of $\mathrm{Fe}$ II/ $\mathrm{Mg}$ II UV lines is one possible abundance indicator.

4. Most of the QSO evolution models predict that $\mathrm{Fe}$ becomes overabundant after $\sim 1$ to $\sim 2$ Gyr by factors of $\sim 2$ to $\sim 10$ relative to solar (i.e., in $[\mathrm{Fe} / \mathrm{O}]$ ). This overabundance could explain the strong Fe II emission observed in QSOs and AGNs.

5. Perhaps the most important result is that the models derived for the QSOs are completely consistent with the evolution expected in massive galactic nuclei. The model that is "typical" of the high-redshift-high-metallicity QSOs, M4, is nearly identical to the models proposed for giant elliptical galaxies (Arimoto \& Yoshii 1987; Matteucci \& Tornambè 1987; Angeletti \& Giannone 1990). These models are, in turn, consistent with the metallicities and evolution derived from observations of ellipticals using population synthesis techniques (Bica 1988; Bica et al. 1988). In M4 the rapid star formation leads to $\sim 85 \%$ of the mass locked up in stars and stellar remnants when the system is only $0.5 \mathrm{Gyr}$ old. The short timescales and greater proportion of high-mass stars compared to the solar neighborhood leads to a metallicity of $\sim 6 Z_{\odot}$ at $0.5 \mathrm{Gyr}$ and nearly $10 Z_{\odot}$ at $1.0 \mathrm{Gyr}$.

The lower line ratios, particularly at low redshifts, require lesser enrichment and metallicities less than a few times solar. These systems might be in an earlier phase of evolution before $Z$ and [N/O] have reached their "final" values. They might 
also be analogous to lower mass ellipticals or to the bulges of disk galaxies, where the evolution is "complete" but lower $Z$ 's are attained. In lower mass ellipticals the lower $Z$ 's are attributed to galactic winds that halt the star formation before the gas is fully processed $(\S 4.4)$. In the bulge of our Galaxy the lower $Z$, compared to giant ellipticals, is attributed to the steeper IMF (Matteucci \& Brocato 1990; Köppen \& Arimoto 1990). Observations of the Galactic bulge reveal a broad metallicity distribution among the stars, peaking at $\sim 2 Z_{\odot}$ and reaching as high as $\sim 10 Z_{\odot}$ (Rich 1988). McWilliams \& Rich (1993) claim these estimates are a factor of $\sim 2$ too high. Nonetheless, the metallicity in the gas is always greater than the mean metallicity in the stars, so the point remains that the gas in the bulge has (or had) $Z>Z_{\odot}$. Models of the bulge evolution indicate that the enrichment occurred in less than $\sim 1 \mathrm{Gyr}$ and that the IMF favored massive stars slightly more than the solar neighborhood (Matteucci \& Brocato 1990; Köppen \& Arimoto 1990). These timescales are comparable to our models M3-M6, and the power-law IMFs applied to the bulge, ranging from $x \approx 1.1$ to 1.35 , are bracketed by M3 and M4 .

Unfortunately, the total mass in gas and stars contributing to the QSO enrichment is unknown. Our results apply strictly to just the broad-line gas, which, according to standard accretion disk models, occupies a region $\lesssim 1$ pc across (Peterson et al. 1991; Peterson \& Gaskell 1991; Clavel et al. 1991; Koratkar \& Gaskell 1991b). The chemical evolution we describe probably involves a stellar population in a larger volume that could include entire galaxies. The similarities with ellipticals suggest that whole galaxies are involved, although the central cores where QSOs reside could reach higher metallicities and evolve more quickly than the rest of the galaxy (cf. Pagel \& Edmunds 1981). In either case, even just the cores of massive galaxies can involve considerable mass. In $\S 4.5$ we showed that a stellar population evolving like M4 would produce peak luminosities $\gtrsim 10^{13}\left(M_{T} / 10^{11} M_{\odot}\right) L_{\odot}$ and $\mathrm{SN}$ rates $\gtrsim 50\left(M_{T} / 10^{11} M_{\odot}\right) \mathrm{yr}^{-1}$. The observed QSOs have luminosities $\left(v L_{v}\right)$ between $\sim 10^{10}$ and almost $10^{15} L_{\odot}$ (Fig. 7). Therefore main-sequence stars controlled by evolution like M4 can rival the observed QSO luminosities if sufficient mass is involved.

Lower mass systems could achieve similarly large luminosities and SN rates if the star formation proceeds more rapidly. The only limitation is that there is enough time for several generations of high mass stars to enrich the gas. We chose the timescales in our models M3-M6 to be essentially the longest allowed by the high-redshift data (assuming $q_{0} \approx \frac{1}{2}$ ). We could have increased the star formation rate $(v)$ by a factor of $\sim 5$ in M4, and made the infall instantaneous, to match the observed line ratios and achieve roughly the same $Z$ 's and $[\mathrm{N} / \mathrm{C}]$ and $[\mathrm{N} / \mathrm{He}]$ abundances in about one-fifth of the time. The resulting peak luminosity would have increased by nearly an order of magnitude for a given mass. Thus an evolving system of mass $\sim 10^{10} M_{\odot}$ could also achieve luminosities $\gtrsim 10^{13} L_{\odot}$ from just the main-sequence stars.

The flux contributions from normal stellar processes are also stressed by the starburst models of AGNs and QSOs (cf. Terlevich 1990; Terlevich et al. 1992). These models attribute all of the light from QSOs to the starburst. Terlevich \& Boyle (1993) recently showed that only $\sim 5 \%$ of the total mass of elliptical galaxies need participate in the bursts to explain the observed luminosities and luminosity function of QSOs with redshift $\gtrsim 2$. However, the evolution discussed here differs from the starburst models because it requires at least several generations of massive stars. The minimum enrichment timescales of $\sim 0.1$ to $\sim 0.2$ Gyr (above) are still several times longer than the bursts discussed by Terlevich \& Boyle (1992). Furthermore, the distribution of line ratios (Fig. 4) implies that the evolution toward high $Z$ occurs before the QSOs become observable. In contrast, the starburst models assume the gas is already enriched when the burst begins (Terlevich et al. 1992).

The important point is that our models require a population of galaxies or galactic nuclei evolving rapidly at redshifts $>2$. The hottest stars will dominate the stellar luminosity, as long there is ongoing star formation, and that luminosity scales linearly with the mass of the evolving population $(\$ 4.5$ and Fig. 9). Spectrophotometric searches for these systems might eventually place limits on their total masses and star formation rates.

We thank J. Baldwin for stimulating discussions on the spectral synthesis and for allowing us to use his spectroscopic data prior to publication. We thank S. Woosley and T. Weaver for helpful advice on the nucleosynthesis in massive stars and for making available their unpublished He core masses. We are grateful to the referee C. M. Gaskell, and to J. Frogel, P. Osmer, F. Matteucci, and R. Terlevich for discussions and comments on the manuscript. We also thank D. Alloin, I. Aretxaga, D. Arnett, R. Cid Fernandez, T. Heckman, P. Padovani, D. Peterson, M. Rees, G. Shields, J. Shields, R. Terlevich, D. Terndrup, and C. Wheeler for helpful discussions. The work of F. H. is supported by NASA grant NAG 5-1645 and by a Columbus Fellowship at the Ohio State University. G. F. is supported by the National Science Foundation through grant AST 90-19692.

\section{REFERENCES}

Abia, C. Canal, R., \& Isern, J. 1991, ApJ, 366, 198

Allen, C. W. 1976, Astrophysical Quantities (3d ed.; London: Athlone) Angeletti, L., \& Giannone, P. 199, A\&A, 234, 53

Arimoto, N., \& Yoshii, Y. 1987, A\&A, 173, 23

Arnett, W. D. 1978, ApJ, 219, 1008

. 1991, in Frontiers of Stellar Evolution, ed. D. L. Lambert (ASP Conf. Ser., 20), 389

Arnett, W. D., Bahcall, J. N., Kirshner, R. P., \& Woosley, S. E. 1989, ARA\&A, 27,629

Arnett, W. D., Schramm, D. N., \& Truran, J. W. 1989, ApJ, 339, L25

Avni, Y., \& Tannanbaum, H. 1985, ApJ, 305, 83

Baldwin, J. A. 1977, ApJ, 214, 679

Baldwin, J. A., Ferland, G., Hamann, F., Phillips, M., Williams, R., \& Wilkes, B. 1983, ApJ, submitted

Baldwin, J. A., Ferland, G. J., Martin, P. G., Corbin, M. R., Cota, S. A., Peterson, B. M. \& Slettebak, A. 1991, ApJ, 374, 580

Baldwin, J. A., \& Netzer, H. 1978, ApJ, 226, 1

Baldwin, J. A., Wampler, E. J., \& Gaskell, C. M. 1989, ApJ, 338, 630
Barbuy, B., \& Grenon, M. 1990, in Bulges of Galaxies (ESO Conf, and Workshop Proc., 35), ed. B. J. Jarvis \& D. M. Terndrup (Garching: ESO), 83 Barthel, P. D., Tytler, D. R., \& Thomson, B. 1990, A\&AS, 82, 339 Barvainis, R. 1990, ApJ, 353, 419

Becker, S. A.. \& Iben, I. 1979, ApJ, 232, 831

Bertelli, G., Bressen, A. G., \& Choisi, C. 1985, A\&A, 150, 33

Bessel, M. S., Sutherland, R. S., \& Ruan, K. 1991, ApJ, 383, L71

Bica, E. 1988, A\&A, 195, 76

Bica, E., Arimoto, N., \& Alloin, D. 1988, A\&A, 202, 8

Burstein, D., \& Heiles, C. 1978, Astrophys. Lett., 19, 69

Carbon, D. F., Barbuy, B., Kraft, R. P., Friel, E. D., \& Suntzeff, N. B. 1987, PASP, 99, 335

Castellani, V., Chieffi, A., \& Straniero, O. 1992, ApJS, 78, 517

Cheng, F. H., Gaskell, C. M., \& Koratkar, A. 1991, ApJ, 370, 487

Clavel, J., et al. 1991, ApJ, 366, 64

Collin-Souffrin, S., Hameury, J. M., \& Joly, M. 1988, A\&A, 205, 19

Cox, D. P. 1972, ApJ, 178, 159

Davidson, K. 1973, ApJ, 181, 1 
Davidson, K. 1977, ApJ, 218, 20

Davidson, K., \& Netzer, H. 1979, Rep. Prog. Phys., 51, 715

Diaz, A. I. 1989, in Evolutionary Phenomena in Galaxies, ed. J. E. Backman \& B. E. J. Pagel (Cambridge: Cambridge Univ. Press), 377

Edmunds, M. G., Greenhow, R. M., Johnson, D., Klückers, V., \& Vila, M. B. 1991, MNRAS, 251, 33P

- Elitzur, M., \& Ferland, G. J. 1986, ApJ, 395, 35

Evans, R., van den Bergh, S., \& McClure, R. D. 1989, ApJ, 345, 752

Ferland, G. J. 1993, HAZY, a Brief Introduction to Cloudy, University of Kentucky Internal Report, in preparation

Ferland, G. J., \& Hamann, F. 1993, in the Nature of Compact Objects in AGN, 33rd Herstmonceux Conference (Cambridge: Cambridge Univ. Press), in press

Ferland, G. J., Peterson, B. M., Horne, K., Welsch, W. F., \& Nahar, S. N. 1992, ApJ, 387, 95

Gaskell, C. M., Shields, G. A., \& Wampler, E. J. 1981, ApJ, 249, 443

Greggio, L., \& Renzini, A. 1983, A\&A, 118, 217

Greggio, L. \& Tosi, M. 1986, A\&AL, 156, L1

Grevesse, N., \& Anders, E. 1989, in Cosmic Abundances of Matter (AIP Conf. Proc. 183), ed. C. I. Waddington (New York: AIP), 1

Hamann, F., \& Ferland, G. J. 1992, ApJ, 391, L53 (HF92)

-.1993a, Rev. Mexicana Astron. Af., in press 1993 b in preparation

Hamann, F., Ferland, G. J., \& Johansson, S. 1993, in preparation

Iben, I. 1967, ARA\&A, 5, 571

Kinney, A. L., Bohlin, R. C., Blades, J. C., \& York, D. G. 1991, ApJS, 75, 645

Kinney, A. L., Rivolo, A. R., \& Koratkar, A. R. 1990, ApJ, 357, 338

Köppen, J., \& Arimoto, N. 1990, A\&A, 240, 22

Koratkar, A. P., \& Gaskell, C. M. 1991a, ApJ, 370, L61 .1991b, ApJS, 75, 719

Kurucz, R. L. 1979, ApJS, 40, 1

Leitherer, C., Gruenwald, R., \& Schmutz, W. 1992, in Physics of Nearby Galaxies, ed. T. X. Thaun, C. Balkowski, \& J. T. T. Van (Paris: Edition Frontières), in press

Lightman, A. P., \& White, T. R. 1988, ApJ, 335, 57

Maeder, A. 1993, A\&A, 120, 113

. 1990, A\&AS, 82,139

Maeder, A., \& Meynet, G. 1987, A\&A, 182, 243

. 1989, A\&A, 210, 155

Mathews, W. G., \& Ferland, G. J. 1987, ApJ, 323, 456 (MF)

Matteucci, F. 1989, in Evolutionary Phenomena in Galaxies, ed. J. E. Backman \& B. E. J. Pagel (Cambridge: Cambridge Univ. Press), 297

1991, in Frontiers of Stellar Evolution, ed. D. L. Lambert (ASP Conf. Ser., 20), 539

Matteucci, F., \& Brocato, E. 1990, ApJ, 365, 539

Matteucci, F., \& François, P. 1989, MNRAS, 239, 885

Matteucci, F., \& Greggio, L. 1986, A\&A, 154, 279

Matteucci, F., \& Tornambè, A 1986, A\&A, 185, 51

McWilliams, A., \& Rich, R. M. 1993, preprint

Netzer, H. 1985, ApJ, 289, 451

Netzer, H., \& Wills, B. J. 1983, ApJ, 275, 445

Nissan, P. E. 1990, in Elements in the Cosmos, ed. M. G. Edmunds \&

R. Terlevich (Cambridge Univ. Press), 110

Nomoto, K., Thielemann, F., \& Yokoi, K. 1984, ApJ, 286, 644

Osmer, P. S. 1980, ApJ, 237, 666

Osmer, P. S., \& Smith, M. G. 1977, ApJ, 213, 607

Osterbrock, D. E. 1977, ApJ, 215, 733

Padovani, P., Burg, R., \& Edelson, R. A. 1990, ApJ, 353, 438

Padovani, P., \& Rafanelli, P. 1988, A\&A, 205, 53

Pagel, B. E. J. 1985, in Production and Distribution of C, N, O Elements, ed. I. J. Danziger, F. Matteucci, \& K. Kjär (Garching: ESO), 155
Pagel, B. E. J. 1989a, in Evolutionary Phenomena in Galaxies, ed. J. E. Backman \& B. E. J. Pagel (Cambridge: Cambridge Univ. Press), 201

1992, in IAU Symp. 149, The Stellar Population of Galaxies, ed. B. Barbuy (Dordrecht: Kluwer), 227

Pagel, B. E. J., \& Edmunds, M. G. 1981, ARA\&A, 19, 77

Pagel, B. E. J., Simonson, E. A., Terlevich, R. J., \& Edmunds, M. G. 1992, MNRAS, 255, 325

Pagel, B. E. J., Terlevich, R., \& Melnick, J. 1986, PASP, 98, 1005

Peimbert, M. 1986, PASP, 98, 1057

Penston, M. 1987, MNRAS, 229, 1P

Peterson, B. M. 1993, PASP, 105, 1084

Peterson, B. M., \& Gaskell, C. M. 1991, ApJ, 368, 152

Peterson, B. M., et al. 1991, ApJ, 368, 119

Phillips, M. M. 1978, ApJS, 38, 187

Press, W. H., Flannery, B. P., Teukolsky, S. A., \& Vetterling, W. T. 1986, Numerical Recipes: The Art of Scientific Computing (Cambridge: Cambridge Univ. Press)

Renzini, A., \& Voli, M. 1981, A\&A, 94, 175

Rich, R. M. 1988, AJ, 95,828

Sanders, D. B., et al. 1989, ApJ, 347, 29

Sargent, W. L. W., Steidel, C. C., \& Boksenberg, A. 1989, ApJS, 69, 703

Scalo, J. M. 1986, Fund. Cosmic Physics, 11, 1 1990, in Windows on Galaxies, ed. G. Fabbiano, J. S. Gallagher, \& A. Renzini (Dordrecht: Kluwer), 125

Schild, H., \& Maeder, A. 1985, A\&A, 143, L7

Schneider, D. P., Schmidt, M., \& Gunn, J. E. 1989a, AJ, 98, 1507 $1989 \mathrm{~b}, \mathrm{AJ}, 98,1951$ 1991 AJ, 102,837

Serrano, A. 1986, PASP, 98, 1066

Shields, G. A. 1976, ApJ, 204, 330

Smecker, T. A., \& Wyse, R. F. G. 1991, ApJ, 372, 448

Stothers, R. B. 1991, ApJ, 383, 820

Stothers, R. B., \& Chin, C. 1991, ApJ, 381, L6

Talbot, R. J., \& Arnett, W. D. 1973, ApJ, 186, 51

Terlevich, R. J. 1990, in Windows on Galaxies, ed. G. Fabbiano, J. Gallagher, \& A. Renzini (Dordrecht: Kluwer), 87

Terlevich, R. J., \& Boyle, B. J. 1983, MNRAS, in press

Terlevich, R. J., Tenorio-Tagle, G., Franco, J., \& Melnick, J. 1992, MNRAS, in press

Thompson, R. I. 1984, ApJ, 283, 165

Tinsley, B. M. 1980, Fund. Cos. Phys., 5, 287

Tomkin, J. \& Lambert, D. L. 1984, ApJ, 279, 220

Truran, J. W. 1987, in Proc. 13th Symp. on Relativistic Astrophys., ed. M. P. Ulmer (Singapore: World Scientific) 430

Uomoto, A. 1984, ApJ, 284, 497

Vader, P. J. 1986a, ApJ, 306, 390

1986b, ApJ, 305, 669 .1987, ApJ, 317, 128

Véron-Cetty, M. P., Véron, P., \& Tarenghi, M. 1983, A\&A, 119, 69

Weaver, T. A., \& Woosley, S. E. 1992, preprint

Wheeler, J. C., \& Harkness, R. P. 1990, Rep. Progr. Phys., 53, 1467

Wheeler, J. C. Sneden, C. \& Truran, J. W. 1989, ARA\& A, 27, 279

Wilkes, B. J. 1984, MNRAS, 207, 73 1986, MNRAS, 218, 331

Wilkes, B., \& Elvis, M. 1987, ApJ, 224, 171

Wills, B. J., Netzer, H., \& Wills, D. 1985, ApJ, 288, 94

Woosley, S. E \& Weaver, T. A 1986, ARA\&A, 24,205

Worthey, G., Farber, S. M., \& Jesús Gonzalez, J. 1992, ApJ, 398, 69

Wyse, R.F. G., \& Gilmore, G. 1988, AJ, 95,1404

Young, P., Sargent, W. L. W., \& Boksenberg, A. 1982, ApJS, 48, 455 